\title{
On the spin susceptibility of noncentrosymmetric superconductors
}

\author{
K. V. Samokhin \\ Department of Physics, Brock University, St.Catharines, Ontario L2S 3A1, Canada
}

(Dated: November 5, 2018)

\begin{abstract}
We calculate the spin susceptibility of a superconductor without inversion symmetry, both in the clean and disordered cases. The susceptibility has a large residual value at zero temperature, which is further enhanced in the presence of scalar impurities.

PACS numbers: 74.20.-z, 74.25.Ha, 74.62.Dh
\end{abstract}

\section{INTRODUCTION}

The discovery of superconductivity in $\mathrm{CePt}_{3} \mathrm{Si}$ (Ref. 1) has renewed interest, both experimental and theoretical, in the properties of superconductors without inversion symmetry. The list of such superconductors has been steadily growing and now also includes UIr (Ref. 2), $\mathrm{CeRhSi}_{3}$ (Ref. 3), $\mathrm{CeIrSi}_{3}$ (Ref. 44), $\mathrm{Y}_{2} \mathrm{C}_{3}$ (Ref. 5), $\mathrm{Li}_{2}\left(\mathrm{Pd}_{1-x}, \mathrm{Pt}_{x}\right)_{3} \mathrm{~B}$ (Ref. 6), and other materials.

A distinctive feature of noncentrosymmetric crystals is that the spin-orbit (SO) coupling qualitatively changes the nature of single-electron states, namely it lifts spin degeneracy and splits energy bands almost everywhere in the Brillouin zone. This has important consequences for superconductivity. In the limit of strong SO coupling, the Cooper pairing between the electrons with opposite momenta occurs only if they are from the same nondegenerate band. According to Ref. 7, this is what happens in $\mathrm{CePt}_{3} \mathrm{Si}$, where the $\mathrm{SO}$ band splitting ranges from $500 \mathrm{~K}$ to $2000 \mathrm{~K}$ and thus exceeds the critical temperature $T_{c}=0.75 \mathrm{~K}$ by orders of magnitude. The same is likely to be the case in other materials, for instance $\mathrm{Li}_{2} \mathrm{Pd}_{3} \mathrm{~B}$ and $\mathrm{Li}_{2} \mathrm{Pt}_{3} \mathrm{~B}$, see Ref. 8 .

The pairing interaction in the strong SO coupling case is most naturally introduced using the exact band states, $, 9,10,11$ which take into account all the effects of the crystal lattice potential and the SO coupling. In the band representation, the superconducting order parameter is represented by a set of complex functions, one for each band, which are coupled, e.g. due to the interband tunneling of the Cooper pairs or the impurity scattering $\stackrel{12}{=}$ Since the bands are nondegenerate, the pairing symmetry is peculiar: While the Pauli principle dictates that each order parameter is an odd function of momentum, the gap symmetry, in particular the location of the gap nodes, is determined by one of the even representations of the crystal point group.

If the band splitting is smaller than the superconducting critical temperature, then the effects of the SO coupling can be treated perturbatively, in particular the pairing Hamiltonian can be constructed using the basis of the pure spinor states unaffected by the SO coupling. This approach to the theory of noncentrosymmetric superconductivity was introduced in Ref. 13 and developed further in Refs. 14, 15 .

One of the most peculiar properties of noncentrosym- metric superconductors is a strongly anisotropic spin susceptibility with a large residual component at zero

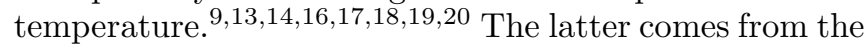
field-induced virtual interband transitions and is almost unchanged across $T_{c}$. Its magnitude depends on the electron structure and is considerably smaller than the normal-state susceptibility, see Sec. IIA below. On the other hand, the experiments in $\mathrm{CePt}_{3} \mathrm{Si}$ (Ref. 21) have detected essentially no change in the Knight shift below $T_{c}$, for all directions of the applied field. One possible explanation is that the relative magnitude of the interband contribution increases due to the combined effect of interactions and peculiarities of the electron structure $\underline{20}$

The goal of this article is to explore an alternative mechanism whereby the residual spin susceptibility is enhanced by disorder. It is well known that the SO impurities play an important role in usual, i.e. centrosymmetric singlet, superconductors, in which experiments also revealed a large residual Knight shift, see, e.g. Ref. 22 and the references therein. This is incompatible with the predictions of the Bardeen-Cooper-Schrieffer (BCS) theory, even when the SO coupling with the crystal lattice potential is taken into account, $\stackrel{23}{=}$ and can be explained by the presence of spin-reversing scattering at the sample boundaries or impurities $\stackrel{24,25,26}{ }$ We calculate the spin susceptibility of a noncentrosymmetric superconductor in the presence of scalar disorder. In contrast to Refs. 13 and 16, we focus on the strong SO coupling limit and employ the band representation of the pairing Hamiltonian.

The article is organized as follows: In Sec. II we calculate the spin susceptibility in the clean case. In Sec. III, we introduce the impurity scattering in the band representation and derive general expressions for the susceptibility in the disordered case. In Sec. IV] we calculate the residual susceptibility in some simple models, in which the effects of impurities can be worked out analytically. We have also included an Appendix with the derivation of the effective SO Hamiltonian for band electrons.

\section{CLEAN CASE}

In a noncentrosymmetric crystal with $\mathrm{SO}$ coupling the electron bands are nondegenerate. The formal reason is that without the inversion operation one cannot, in general, have two orthogonal degenerate Bloch states at the 
same wave vector $\boldsymbol{k}$. In the limit of zero SO coupling there is an additional symmetry in the system - the invariance with respect to arbitrary rotations in spin space - which preserves two-fold degeneracy of the bands. Let us consider a single band of electrons with the dispersion given by $\epsilon(\boldsymbol{k})$, and turn on the SO coupling with the crystal lattice. The system Hamiltonian can be written in the following form:

$$
H_{0}=\sum_{\boldsymbol{k}, \alpha \beta}\left[\epsilon(\boldsymbol{k}) \delta_{\alpha \beta}+\gamma(\boldsymbol{k}) \boldsymbol{\sigma}_{\alpha \beta}\right] a_{\boldsymbol{k} \alpha}^{\dagger} a_{\boldsymbol{k} \beta} .
$$

Here $\alpha, \beta=\uparrow, \downarrow$ label the spin states, $\boldsymbol{\sigma}$ are the Pauli matrices, and the sum over $\boldsymbol{k}$ is restricted to the first Brillouin zone. The "bare" band dispersion satisfies $\epsilon(-\boldsymbol{k})=\epsilon(\boldsymbol{k}), \epsilon\left(g^{-1} \boldsymbol{k}\right)=\epsilon(\boldsymbol{k})$, where $g$ is any operation from the point group $\mathbb{G}$ of the crystal. The SO coupling is described by the pseudovector function $\gamma(\boldsymbol{k})$, which has the following symmetry properties: $\gamma(\boldsymbol{k})=-\gamma(-\boldsymbol{k})$, $g \gamma\left(g^{-1} \boldsymbol{k}\right)=\gamma(\boldsymbol{k})$. The derivation of Eq. (1) is outlined in the Appendix.

For the tetragonal group $\mathbb{G}=\mathbf{C}_{4 v}$, which describes the point symmetry of $\mathrm{CePt}_{3} \mathrm{Si}, \mathrm{CeRhSi}_{3}$ and $\mathrm{CeIrSi}_{3}$, the SO coupling has the following form:

$$
\boldsymbol{\gamma}(\boldsymbol{k})=\gamma_{\perp}\left[\phi_{E, u}(\boldsymbol{k}) \times \hat{z}\right]+\gamma_{\|} \phi_{A_{2}, u}(\boldsymbol{k}) \hat{z} .
$$

Here $\gamma_{\perp}$ and $\gamma_{\|}$are constants, and $\phi_{E, u}$ and $\phi_{A_{2}, u}$ are the odd basis functions of the irreducible representations $E$ (two-dimensional) and $A_{2}$ (one-dimensional), respectively. The representative polynomial expressions for the basis functions are $\phi_{E, u}(\boldsymbol{k}) \propto\left(k_{x}, k_{y}\right)$ and $\phi_{A_{2}, u}(\boldsymbol{k}) \propto$ $k_{x} k_{y} k_{z}\left(k_{x}^{2}-k_{y}^{2}\right)$. A particular two-dimensional case of Eq. (2) with $\boldsymbol{\gamma}(\boldsymbol{k})=\gamma_{\perp}(\boldsymbol{k} \times \hat{z})$ is known as the Rashba model ${ }^{27}$ and has been extensively used to study the effects of SO coupling in semiconductor heterostructures. For the cubic group $\mathbb{G}=\mathbf{O}$, which describes the point symmetry of $\mathrm{Li}_{2}\left(\mathrm{Pd}_{1-x}, \mathrm{Pt}_{x}\right)_{3} \mathrm{~B}$, the $\mathrm{SO}$ coupling has the form

$$
\gamma(\boldsymbol{k})=\gamma_{0} \phi_{F_{1}, u}(\boldsymbol{k}),
$$

where $\phi_{F_{1}, u}$ are the odd basis functions of the vector representation $F_{1}$, e.g. $\phi_{F_{1}, u}(\boldsymbol{k}) \propto\left(k_{x}, k_{y}, k_{z}\right)$.

The Hamiltonian (1) is diagonalized by a unitary transformation:

$$
a_{\boldsymbol{k} \alpha}=\sum_{\lambda= \pm} u_{\alpha \lambda}(\boldsymbol{k}) c_{\boldsymbol{k} \lambda}
$$

where

$$
\begin{aligned}
& u_{\uparrow \lambda}(\boldsymbol{k})=e^{i \theta_{\lambda}} \sqrt{\frac{|\gamma|+\lambda \gamma_{z}}{2|\gamma|}}, \\
& u_{\downarrow \lambda}(\boldsymbol{k})=\lambda e^{i \theta_{\lambda}} \frac{\gamma_{x}+i \gamma_{y}}{\sqrt{2|\gamma|\left(|\gamma|+\lambda \gamma_{z}\right)}},
\end{aligned}
$$

and $\theta_{\lambda}$ are arbitrary (in general $\boldsymbol{k}$-dependent) phases. The free-electron Hamiltonian then becomes

$$
H_{0}=\sum_{\boldsymbol{k}} \sum_{\lambda= \pm} \xi_{\lambda}(\boldsymbol{k}) c_{\boldsymbol{k} \lambda}^{\dagger} c_{\boldsymbol{k} \lambda}
$$

where $\xi_{\lambda}(\boldsymbol{k})=\epsilon(\boldsymbol{k})+\lambda|\gamma(\boldsymbol{k})|=\xi_{\lambda}(-\boldsymbol{k})$ describes the quasiparticle dispersion in the $\lambda$ th band. Thus the SO coupling lifts the spin degeneracy of the electron bands, so that the Fermi surface consists of two pieces defined by the equations $\xi_{ \pm}(\boldsymbol{k})=0$. The magnitude of the band splitting is given by $2|\gamma(\boldsymbol{k})|$ and might vanish, for symmetry reasons, along some directions or at some isolated points in the Brillouin zone. We assume that the band structure is such that the zeros of $\gamma(\boldsymbol{k})$ are not located on the Fermi surface. This is the case in the model (3), and also in the model (2) if the Fermi surface is a cylinder around the $z$-axis.

The Zeeman coupling of the electron spins with an external magnetic field is described by

$$
\begin{aligned}
H_{Z} & =-\mu_{B} \boldsymbol{B} \sum_{\boldsymbol{k}, \alpha \beta} \boldsymbol{\sigma}_{\alpha \beta} a_{\boldsymbol{k} \alpha}^{\dagger} a_{\boldsymbol{k} \beta} \\
& =-\boldsymbol{B} \sum_{\boldsymbol{k}, \lambda \lambda^{\prime}} \boldsymbol{m}_{\lambda \lambda^{\prime}}(\boldsymbol{k}) c_{\boldsymbol{k} \lambda}^{\dagger} c_{\boldsymbol{k} \lambda^{\prime}},
\end{aligned}
$$

where $\mu_{B}$ is the Bohr magneton. The components of $\hat{\boldsymbol{m}}(\boldsymbol{k})=\mu_{B} \hat{u}^{\dagger}(\boldsymbol{k}) \hat{\boldsymbol{\sigma}} \hat{u}(\boldsymbol{k})$ have the following form:

$$
\begin{aligned}
& \hat{m}_{x}=\mu_{B}\left(\begin{array}{cc}
\hat{\gamma}_{x} & -e^{-i \tilde{\theta} \frac{\gamma_{x} \hat{\gamma}_{z}+i \gamma_{y}}{\gamma_{\perp}}} \\
-e^{i \tilde{\theta} \frac{\gamma_{x} \hat{\gamma}_{z}-i \gamma_{y}}{\gamma_{\perp}}} & -\hat{\gamma}_{x}
\end{array}\right), \\
& \hat{m}_{y}=\mu_{B}\left(\begin{array}{cc}
\hat{\gamma}_{y} & -e^{-i \tilde{\theta} \frac{\gamma_{y} \hat{\gamma}_{z}-i \gamma_{x}}{\gamma_{\perp}}} \\
-e^{i \tilde{\theta} \frac{\gamma_{y} \hat{\gamma}_{z}+i \gamma_{x}}{\gamma_{\perp}}} & -\hat{\gamma}_{y}
\end{array}\right), \\
& \hat{m}_{z}=\mu_{B}\left(\begin{array}{cc}
\hat{\gamma}_{z} & e^{-i \tilde{\theta} \frac{\gamma_{\perp}}{\gamma}} \\
e^{i \tilde{\theta} \frac{\gamma_{\perp}}{\gamma}} & -\hat{\gamma}_{z}
\end{array}\right),
\end{aligned}
$$

where $\hat{\gamma}=\gamma /|\gamma|, \gamma_{\perp}=\sqrt{\gamma_{x}^{2}+\gamma_{y}^{2}}$, and $\tilde{\theta}=\theta_{+}-\theta_{-}$. The expectation value of the spin magnetic moment of an electron from the $\lambda$ th band with the wave vector $\boldsymbol{k}$ is $\boldsymbol{m}_{\lambda}(\boldsymbol{k})=\lambda \mu_{B} \hat{\boldsymbol{\gamma}}(\boldsymbol{k})$. We shall see that, although the interband matrix elements of $\hat{\boldsymbol{m}}(\boldsymbol{k})$ contain the arbitrary phases of the Bloch spinors, those will not affect observable quantities.

Finally, we introduce the pairing interaction between electrons in the Cooper channel, using the basis of the exact eigenstates of the noninteracting problem:

$$
\begin{array}{r}
H_{i n t}=\frac{1}{2 \mathcal{V}} \sum_{\boldsymbol{k} \boldsymbol{k}^{\prime} \boldsymbol{q}} \sum_{\lambda \lambda^{\prime}} V_{\lambda \lambda^{\prime}}\left(\boldsymbol{k}, \boldsymbol{k}^{\prime}\right) c_{\boldsymbol{k}+\boldsymbol{q} / 2, \lambda}^{\dagger} c_{-\boldsymbol{k}+\boldsymbol{q} / 2, \lambda}^{\dagger} \\
\times c_{-\boldsymbol{k}^{\prime}+\boldsymbol{q} / 2, \lambda^{\prime}} c_{\boldsymbol{k}^{\prime}+\boldsymbol{q} / 2, \lambda^{\prime}},
\end{array}
$$

where $\mathcal{V}$ is the system volume. The pairing potential satisfies the relations $V_{\lambda \lambda^{\prime}}\left(-\boldsymbol{k}, \boldsymbol{k}^{\prime}\right)=-V_{\lambda \lambda^{\prime}}\left(\boldsymbol{k}, \boldsymbol{k}^{\prime}\right)=$ $V_{\lambda \lambda^{\prime}}\left(\boldsymbol{k},-\boldsymbol{k}^{\prime}\right)$, which follow from the anti-commutation of the Fermi operators. The diagonal elements of the matrix $\hat{V}$ describe the intraband Cooper pairing, while the off-diagonal ones correspond to the pair scattering from one band to the other. We assume, in the spirit of the BCS theory, that the pairing interaction is nonzero only inside the thin shells of width $\varepsilon_{c}$ in the vicinity of the Fermi surfaces, i.e. when $\left|\xi_{\lambda}(\boldsymbol{k})\right|,\left|\xi_{\lambda^{\prime}}\left(\boldsymbol{k}^{\prime}\right)\right| \leq \varepsilon_{c}$. We 
further assume that it can be represented in a factorized form: $V_{\lambda \lambda^{\prime}}\left(\boldsymbol{k}, \boldsymbol{k}^{\prime}\right)=t_{\lambda}(\boldsymbol{k}) t_{\lambda^{\prime}}^{*}\left(\boldsymbol{k}^{\prime}\right) \tilde{V}_{\lambda \lambda^{\prime}}\left(\boldsymbol{k}, \boldsymbol{k}^{\prime}\right)$, where $t_{\lambda}(\boldsymbol{k})=-t_{\lambda}(-\boldsymbol{k})$ are nontrivial phase factors which appear in the expression for the time-reversal operation for nondegenerate bands: $K|\boldsymbol{k} \lambda\rangle=t_{\lambda}(\boldsymbol{k})|-\boldsymbol{k}, \lambda\rangle, \underline{,, 10}$ and

$$
\tilde{V}_{\lambda \lambda^{\prime}}\left(\boldsymbol{k}, \boldsymbol{k}^{\prime}\right)=-V_{\lambda \lambda^{\prime}} \sum_{a=1}^{d_{\Gamma}} \phi_{\lambda, a}(\boldsymbol{k}) \phi_{\lambda^{\prime}, a}^{*}\left(\boldsymbol{k}^{\prime}\right)
$$

is invariant under the point group operations: $\tilde{V}_{\lambda \lambda^{\prime}}\left(g^{-1} \boldsymbol{k}, g^{-1} \boldsymbol{k}^{\prime}\right)=\tilde{V}_{\lambda \lambda^{\prime}}\left(\boldsymbol{k}, \boldsymbol{k}^{\prime}\right)$. The coupling constants $V_{\lambda \lambda^{\prime}}$ form a symmetric positive-definite $2 \times 2$ matrix, and $\phi_{\lambda, a}(\boldsymbol{k})$ are even basis functions of an

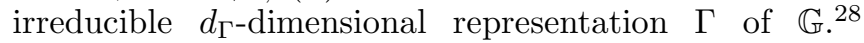
While $\phi_{+, a}(\boldsymbol{k})$ and $\phi_{-, a}(\boldsymbol{k})$ have the same symmetry, their momentum dependence does not have to be exactly the same. The basis functions are nonzero only inside the BCS shells and are normalized: $\left\langle\phi_{\lambda, a}^{*} \phi_{\lambda, b}\right\rangle_{\lambda}=\delta_{a b}$, where the angular brackets denote the averaging over the Fermi surface in the $\lambda$ th band.

Treating the pairing interaction (9) in the mean-field approximation, one introduces the superconducting order parameters, which in the uniform case considered here depend only on $\boldsymbol{k}$ and have the form $\Delta_{\lambda}(\boldsymbol{k})=$ $t_{\lambda}(\boldsymbol{k}) \tilde{\Delta}_{\lambda}(\boldsymbol{k})$, where $\tilde{\Delta}_{\lambda}(\boldsymbol{k})=\tilde{\Delta}_{\lambda}(-\boldsymbol{k})$ can be represented as follows:

$$
\tilde{\Delta}_{\lambda}(\boldsymbol{k})=\sum_{a} \eta_{\lambda, a} \phi_{\lambda, a}(\boldsymbol{k})
$$

Thus our system is formally equivalent to a two-band superconductor, in which the order parameter has $2 d_{\Gamma}$ components given by the expansion coefficients $\eta_{\lambda, a}$. The order parameter components depend on temperature and vanish in the normal state.

\section{A. Spin susceptibility}

Writing the Zeeman Hamiltonian (7) in the form $H_{Z}=$ $-\mathcal{M} \boldsymbol{B}$, where $\mathcal{M}$ is the operator of the total spin magnetic moment of electrons, we define the magnetization as $\boldsymbol{M}=\mathcal{V}^{-1}\langle\mathcal{M}\rangle$ (the angular brackets denote the thermodynamic average). In a weak field, $M_{i}=\sum_{j} \chi_{i j} B_{j}$, where $\chi_{i j}$ is the spin susceptibility tensor.

Introducing the four-component Nambu operators in the band representation: $C_{\boldsymbol{k}}=\left(c_{\boldsymbol{k}+}, c_{\boldsymbol{k}-}, c_{-\boldsymbol{k},+}^{\dagger}, c_{-\boldsymbol{k},+}^{\dagger}\right)^{T}$, we combine the normal and anomalous Green's functions ${ }^{29}$ into a $4 \times 4$ matrix Green's function

$$
\mathcal{G}\left(\boldsymbol{k}_{1}, \boldsymbol{k}_{2} ; \tau\right)=-\left\langle T_{\tau} C_{\boldsymbol{k}_{1}}(\tau) C_{\boldsymbol{k}_{2}}^{\dagger}(0)\right\rangle .
$$

In the clean system, the Green's function is diagonal in momentum:

$$
\mathcal{G}\left(\boldsymbol{k}, \omega_{n}\right)=\left(\begin{array}{cc}
\hat{G}\left(\boldsymbol{k}, \omega_{n}\right) & -\hat{F}\left(\boldsymbol{k}, \omega_{n}\right) \\
-\hat{F}^{\dagger}\left(\boldsymbol{k}, \omega_{n}\right) & -\hat{G}^{T}\left(-\boldsymbol{k},-\omega_{n}\right)
\end{array}\right) .
$$

Here $\omega_{n}=(2 n+1) \pi T$ is the fermionic Matsubara frequency (in our units $k_{B}=1$ ), and the hats here denote $2 \times 2$ matrices in the band space. In the thermodynamic limit $\mathcal{V} \rightarrow \infty$ the magnetization can be expressed in terms of the Green's functions as follows:

$$
\boldsymbol{M}=T \sum_{n} \int \frac{d^{3} \boldsymbol{k}}{(2 \pi)^{3}} \operatorname{tr} \hat{\boldsymbol{m}}(\boldsymbol{k}) \hat{G}\left(\boldsymbol{k}, \omega_{n}\right),
$$

where the matrices $\hat{\boldsymbol{m}}$ are given by Eqs. (8).

In the presence of magnetic field we have $\mathcal{G}\left(\boldsymbol{k}, \omega_{n}\right)=$ $\left[\mathcal{G}_{0}^{-1}\left(\boldsymbol{k}, \omega_{n}\right)-\Sigma_{Z}(\boldsymbol{k})\right]^{-1}$, where

$$
\mathcal{G}_{0}^{-1}\left(\boldsymbol{k}, \omega_{n}\right)=\left(\begin{array}{cc}
i \omega_{n}-\hat{\xi}(\boldsymbol{k}) & -\hat{\Delta}(\boldsymbol{k}) \\
-\hat{\Delta}^{\dagger}(\boldsymbol{k}) & i \omega_{n}+\hat{\xi}(\boldsymbol{k})
\end{array}\right),
$$

$\Delta_{\lambda \lambda^{\prime}}(\boldsymbol{k})=\delta_{\lambda \lambda^{\prime}} t_{\lambda}(\boldsymbol{k}) \tilde{\Delta}_{\lambda}(\boldsymbol{k})$, and the Zeeman coupling is described by

$$
\Sigma_{Z}(\boldsymbol{k})=\left(\begin{array}{cc}
-\hat{\boldsymbol{m}}(\boldsymbol{k}) \boldsymbol{B} & 0 \\
0 & \hat{\boldsymbol{m}}^{T}(-\boldsymbol{k}) \boldsymbol{B}
\end{array}\right) .
$$

Expanding $\mathcal{G}$ in powers of $\boldsymbol{B}$, we obtain:

$$
\chi_{i j}=-T \sum_{n} \int \frac{d^{3} \boldsymbol{k}}{(2 \pi)^{3}} \operatorname{tr}\left(\hat{m}_{i} \hat{G} \hat{m}_{j} \hat{G}-\hat{m}_{i} \hat{F} \hat{\bar{m}}_{j} \hat{F}^{\dagger}\right) \text {, }
$$

where $\hat{\overline{\boldsymbol{m}}}(\boldsymbol{k})=\hat{\boldsymbol{m}}^{T}(-\boldsymbol{k})$. The Green's functions here are calculated at zero field and have the following form:

$$
\begin{aligned}
& G_{\lambda \lambda^{\prime}}\left(\boldsymbol{k}, \omega_{n}\right)=\delta_{\lambda \lambda^{\prime}} G_{\lambda}\left(\boldsymbol{k}, \omega_{n}\right), \\
& F_{\lambda \lambda^{\prime}}\left(\boldsymbol{k}, \omega_{n}\right)=\delta_{\lambda \lambda^{\prime}} t_{\lambda}(\boldsymbol{k}) \tilde{F}_{\lambda}\left(\boldsymbol{k}, \omega_{n}\right), \\
& F_{\lambda \lambda^{\prime}}^{\dagger}\left(\boldsymbol{k}, \omega_{n}\right)=\delta_{\lambda \lambda^{\prime}} t_{\lambda}^{*}(\boldsymbol{k}) \tilde{F}_{\lambda}^{*}\left(\boldsymbol{k}, \omega_{n}\right),
\end{aligned}
$$

where

$$
\begin{aligned}
& G_{\lambda}\left(\boldsymbol{k}, \omega_{n}\right)=-\frac{i \omega_{n}+\xi_{\lambda}(\boldsymbol{k})}{\omega_{n}^{2}+\xi_{\lambda}^{2}(\boldsymbol{k})+\left|\tilde{\Delta}_{\lambda}(\boldsymbol{k})\right|^{2}}, \\
& \tilde{F}_{\lambda}\left(\boldsymbol{k}, \omega_{n}\right)=\frac{\tilde{\Delta}_{\lambda}(\boldsymbol{k})}{\omega_{n}^{2}+\xi_{\lambda}^{2}(\boldsymbol{k})+\left|\tilde{\Delta}_{\lambda}(\boldsymbol{k})\right|^{2}} .
\end{aligned}
$$

Inserting the expressions (8) for the electron magnetic moment in Eq. (17) and using the identities

$$
\begin{aligned}
& \bar{m}_{i, \lambda \lambda^{\prime}}=-t_{\lambda}^{*} t_{\lambda^{\prime}} m_{i, \lambda \lambda^{\prime}}, \\
& m_{i,++} m_{j,++}=m_{i,--} m_{j,--}=\mu_{B}^{2} \hat{\gamma}_{i} \hat{\gamma}_{j}, \\
& m_{i,+-} m_{j,-+}=\mu_{B}^{2}\left(\delta_{i j}-\hat{\gamma}_{i} \hat{\gamma}_{j}+i e_{i j k} \hat{\gamma}_{k}\right),
\end{aligned}
$$

we find that the susceptibility tensor can be represented in the following form:

$$
\chi_{i j}=\sum_{\lambda= \pm} \chi_{i j}^{\lambda}+\tilde{\chi}_{i j}
$$

Here

$$
\begin{aligned}
\chi_{i j}^{\lambda} & =-\mu_{B}^{2} T \sum_{n} \int \frac{d^{3} \boldsymbol{k}}{(2 \pi)^{3}} \hat{\gamma}_{i} \hat{\gamma}_{j}\left(G_{\lambda}^{2}+\left|\tilde{F}_{\lambda}\right|^{2}\right) \\
& =\mu_{B}^{2} N_{\lambda}\left\langle\hat{\gamma}_{i} \hat{\gamma}_{j} Y_{\lambda}\right\rangle_{\lambda}
\end{aligned}
$$


are the intraband contributions, which are determined by the thermally-excited quasiparticles near the Fermi surfaces, $N_{\lambda}$ is the density of states in the $\lambda$ th band, and

$$
Y_{\lambda}(\boldsymbol{k}, T)=\frac{1}{2 T} \int_{0}^{\infty} \frac{d \xi}{\cosh ^{2}\left(\sqrt{\xi^{2}+\left|\tilde{\Delta}_{\lambda}(\boldsymbol{k})\right|^{2}} / 2 T\right)}
$$

is the angle-resolved Yosida function.

The last term in Eq. (21) is

$$
\begin{array}{r}
\tilde{\chi}_{i j}=-2 \mu_{B}^{2} T \sum_{n} \int \frac{d^{3} \boldsymbol{k}}{(2 \pi)^{3}}\left(\delta_{i j}-\hat{\gamma}_{i} \hat{\gamma}_{j}\right) \\
\times\left(G_{+} G_{-}+\operatorname{Re} \tilde{F}_{+}^{*} \tilde{F}_{-}\right) .
\end{array}
$$

In the normal state this becomes

$$
\tilde{\chi}_{i j}=-\mu_{B}^{2} \int \frac{d^{3} \boldsymbol{k}}{(2 \pi)^{3}} \frac{\delta_{i j}-\hat{\gamma}_{i} \hat{\gamma}_{j}}{|\gamma|}\left[f\left(\xi_{+}\right)-f\left(\xi_{-}\right)\right],
$$

where $f(\epsilon)=\left(e^{\epsilon / T}+1\right)^{-1}$ is the Fermi-Dirac distribution function. In contrast to the intraband susceptibilities $\chi_{i j}^{\lambda}$, which depend only on the quasiparticle properties in the vicinity of the Fermi surfaces, $\tilde{\chi}_{i j}$ is determined by all quasiparticles in the momentum-space shell "sandwiched" between the Fermi surfaces. We therefore call this the interband contribution (its physical origin is discussed in Sec. IIB below). A straightforward calculation shows that even at $T=0$

$$
\frac{\left.\tilde{\chi}_{i j}\right|_{\tilde{\Delta} \neq 0}-\left.\tilde{\chi}_{i j}\right|_{\tilde{\Delta}=0}}{\left.\tilde{\chi}_{i j}\right|_{\tilde{\Delta}=0}} \sim \frac{|\tilde{\Delta}|^{2}}{|\gamma|^{2}} \ln \frac{|\gamma|}{|\tilde{\Delta}|} \ll 1,
$$

which means that $\tilde{\chi}_{i j}$ is almost unchanged when the system undergoes a phase transition in which only the electrons near the Fermi surface are affected.

Thus we arrive at the following expression for the spin susceptibility of a clean superconductor:

$$
\chi_{i j}=\tilde{\chi}_{i j}+\mu_{B}^{2} N_{F} \sum_{\lambda} \rho_{\lambda}\left\langle\hat{\gamma}_{i} \hat{\gamma}_{j} Y_{\lambda}\right\rangle_{\lambda},
$$

where $\tilde{\chi}_{i j}$ is given by Eq. (25), $N_{F}=\left(N_{+}+N_{-}\right) / 2$, and $\rho_{\lambda}=N_{\lambda} / N_{F}$. In the normal state, $Y_{\lambda}=1$ and

$$
\chi_{N, i j}=\tilde{\chi}_{i j}+\mu_{B}^{2} N_{F} \sum_{\lambda} \rho_{\lambda}\left\langle\hat{\gamma}_{i} \hat{\gamma}_{j}\right\rangle_{\lambda} .
$$

At zero temperature, there is no excitations $\left(Y_{\lambda}=0\right)$ and the intraband contributions are absent, but the susceptibility still attains a nonzero value given by $\tilde{\chi}_{i j}$. The temperature dependence of the susceptibility in the superconducting state at $0<T \leq T_{c}$ is almost entirely determined by the intraband terms, with the low-temperature behavior depending crucially on the magnitude of the SO coupling at the gap nodes ${ }^{19}$ While in the fully gapped case the intraband susceptibility is exponentially small in all directions, in the presence of the lines of nodes it is proportional to either $T$ or $T^{3}$, depending on whether or

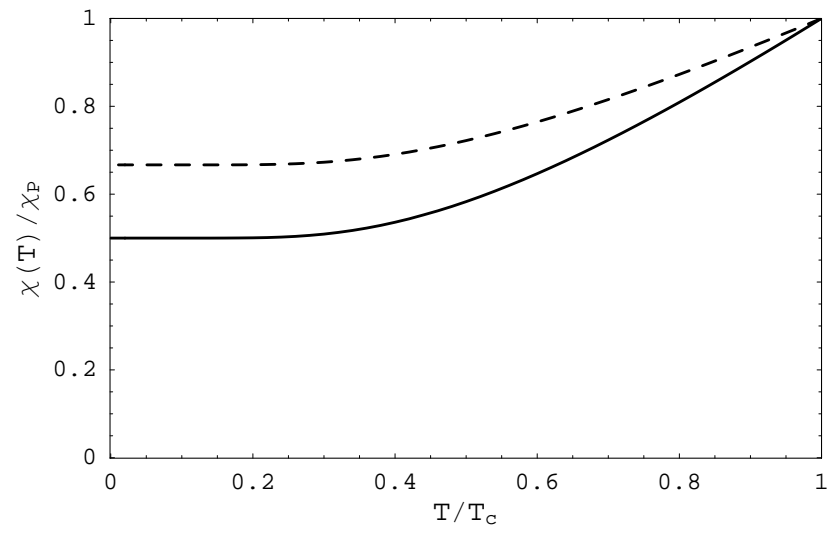

FIG. 1: The temperature dependence of the transverse components of the susceptibility for the $2 \mathrm{D}$ model (the solid line), and of all three components for the 3D model (the dashed line). The $\chi_{z z}$ component in the $2 \mathrm{D}$ case is temperatureindependent.

not the zeros of $\tilde{\Delta}_{\lambda}(\boldsymbol{k})$ coincide with those of $\gamma(\boldsymbol{k})$, see Ref. 19 for details.

Further steps depend on the pairing symmetry and the electron structure of the superconductor. Let us evaluate the expression (26) in the case when the SO coupling is small compared to the Fermi energy, i.e. $|\gamma| \ll \epsilon_{F}$, and the gaps in both bands are isotropic and have the same magnitude. It is legitimate to neglect the difference between the densities of states in the two bands: $\rho_{+}=$ $\rho_{-}=1$, and we also have $Y_{+}(\boldsymbol{k}, T)=Y_{-}(\boldsymbol{k}, T)=Y(T)$. The interband contribution (25) is reduced to

$$
\tilde{\chi}_{i j}=2 \mu_{B}^{2} N_{F}\left(\delta_{i j}-\left\langle\hat{\gamma}_{i} \hat{\gamma}_{j}\right\rangle_{F}\right)
$$

where $\langle(\ldots)\rangle_{F}$ denotes the average over the Fermi surface defined by the equation $\epsilon(\boldsymbol{k})=0$. The normalstate susceptibility is isotropic: $\chi_{N, i j}=\chi_{P} \delta_{i j}$, where $\chi_{P}=2 \mu_{B}^{2} N_{F}$ is the Pauli susceptibility, while in the superconducting state

$$
\chi_{i j}=\tilde{\chi}_{i j}+2 \mu_{B}^{2} N_{F}\left\langle\hat{\gamma}_{i} \hat{\gamma}_{j}\right\rangle_{F} Y(T) .
$$

Suppose the Fermi surface is a cylinder around the $z$ axis and the SO coupling is described by the Rashba expression: $\boldsymbol{\gamma}(\boldsymbol{k})=\gamma_{\perp}(\boldsymbol{k} \times \hat{z})$. We will refer to this as the two-dimensional (2D) model. The susceptibility tensor is diagonal, with

$$
\chi_{x x}=\chi_{y y}=\frac{1}{2}[1+Y(T)] \chi_{P}, \quad \chi_{z z}=\chi_{P} .
$$

For a spherical Fermi surface in a cubic crystal with the SO coupling described by $\boldsymbol{\gamma}(\boldsymbol{k})=\gamma_{0} \boldsymbol{k}$ [the threedimensional (3D) model], the susceptibility tensor is given by

$$
\chi_{x x}=\chi_{y y}=\chi_{z z}=\frac{1}{3}[2+Y(T)] \chi_{P} .
$$


The temperature dependence of $\chi_{i j}$ for the 2D and 3D models is sketched in Fig. 1. In both cases the susceptibility at $T=0$ is nonzero but still considerably less than in the normal state.

\section{B. Origin of the residual susceptibility}

The mean-field pairing Hamiltonian in the band representation is given by $H_{s c}=$ $(1 / 2) \sum_{\boldsymbol{k}, \lambda}\left[\Delta_{\lambda}(\boldsymbol{k}) c_{\boldsymbol{k} \lambda}^{\dagger} c_{-\boldsymbol{k}, \lambda}^{\dagger}+\right.$ H.c. $] . \quad$ This can be transformed into the spin representation using Eqs. (41): $H_{s c}=(1 / 2) \sum_{\boldsymbol{k}, \alpha \beta}\left[\Delta_{\alpha \beta}(\boldsymbol{k}) a_{\boldsymbol{k} \alpha}^{\dagger} a_{-\boldsymbol{k}, \beta}^{\dagger}+\right.$ H.c. $]$, where

$$
\begin{aligned}
\Delta_{\alpha \beta}(\boldsymbol{k}) & =\sum_{\lambda} u_{\alpha \lambda}(\boldsymbol{k}) \Delta_{\lambda}(\boldsymbol{k}) u_{\beta \lambda}(-\boldsymbol{k}) \\
& =-\left[\hat{u}(\boldsymbol{k}) \hat{\tilde{\Delta}}(\boldsymbol{k}) \hat{u}^{\dagger}(\boldsymbol{k})\left(i \hat{\sigma}_{2}\right)\right]_{\alpha \beta} .
\end{aligned}
$$

To obtain this, we used the identity $u_{\beta \lambda}(-\boldsymbol{k})=$ $t_{\lambda}^{*}(\boldsymbol{k}) \sum_{\gamma}\left(i \sigma_{2}\right)_{\beta \gamma} u_{\gamma \lambda}^{*}(\boldsymbol{k})$, which follows from the definition of $t_{\lambda}(\boldsymbol{k})$. The next step is to write $\hat{\tilde{\Delta}}=\left(\tilde{\Delta}_{+}+\tilde{\Delta}_{-}\right) \hat{\tau}_{0} / 2+$ $\left(\tilde{\Delta}_{+}-\tilde{\Delta}_{-}\right) \hat{\tau}_{3} / 2$, where $\hat{\tau}$ are the Pauli matrices. Inserting this in Eq. (32) and using

$$
\hat{u}(\boldsymbol{k}) \hat{\tau}_{3} \hat{u}^{\dagger}(\boldsymbol{k})=\hat{\gamma}(\boldsymbol{k}) \hat{\boldsymbol{\tau}}
$$

we obtain the order parameter in the spin representation as follows:

$$
\Delta_{\alpha \beta}(\boldsymbol{k})=\psi(\boldsymbol{k})\left(i \hat{\sigma}_{2}\right)_{\alpha \beta}+\boldsymbol{d}(\boldsymbol{k})\left(i \hat{\boldsymbol{\sigma}} \hat{\sigma}_{2}\right)_{\alpha \beta},
$$

where

$$
\psi(\boldsymbol{k})=-\frac{\tilde{\Delta}_{+}(\boldsymbol{k})+\tilde{\Delta}_{-}(\boldsymbol{k})}{2}
$$

is the spin-singlet component, and

$$
\boldsymbol{d}(\boldsymbol{k})=-\frac{\tilde{\Delta}_{+}(\boldsymbol{k})-\tilde{\Delta}_{-}(\boldsymbol{k})}{2} \hat{\gamma}(\boldsymbol{k})
$$

is the spin-triplet component. 30 In agreement with the results of Ref. 14, only one component of the triplet order parameter survives (is "protected") in the limit of a large SO band splitting.

We see that the residual susceptibility is not related to the presence of the spin-triplet order parameter: Expression (36) vanishes if the both gaps are the same, but the residual susceptibility is still nonzero and given by Eq. (25). The origin of the residual susceptibility can be understood using the following argument 17 For a given wave vector $\boldsymbol{k}$, there are two electron states $|\boldsymbol{k}, \pm\rangle$ with energies $\xi_{ \pm}(\boldsymbol{k})$, in which the expectation values of the spin magnetic moment are $\boldsymbol{m}_{ \pm}(\boldsymbol{k})= \pm \mu_{B} \hat{\boldsymbol{\gamma}}(\boldsymbol{k})$. Due to the presence of the projector $\delta_{i j}-\hat{\gamma}_{i} \hat{\gamma}_{j}$, only the component of $\boldsymbol{B}$ which is perpendicular to $\boldsymbol{m}_{ \pm}(\boldsymbol{k})$ contributes to the susceptibility (25). Calculating the energies of the states $|\boldsymbol{k}, \pm\rangle$ in the second order of the perturbation theory with the help of the identity (20), we obtain:

$$
\delta \xi_{\lambda}^{(2)}(\boldsymbol{k})=\lambda \mu_{B}^{2} \sum_{i, j} B_{i} B_{j} \frac{\delta_{i j}-\hat{\gamma}_{i} \hat{\gamma}_{j}}{2|\gamma|}
$$

To find the total energy shift, we sum the contributions from all $\boldsymbol{k}$ and both bands, with the weights given by the Fermi-Dirac distribution. In this way we recover Eq. (25). Thus the interband susceptibility appears, similarly to the Van Vleck paramagnetism of atoms and solids, in the second order of the perturbation theory, due to the field-induced virtual transitions between the SO split electron bands.

\section{EFFECTS OF IMPURITIES}

The effect of scalar impurities on the spin susceptibility in the superconducting state is described by the Hamiltonian $H=H_{0}+H_{Z}+H_{i n t}+H_{i m p}$, where the first three terms are given by Eqs. (6), (7) and (9), and

$$
H_{i m p}=\int d^{3} \boldsymbol{r} \sum_{\alpha} U_{i m p}(\boldsymbol{r}) \psi_{\alpha}^{\dagger}(\boldsymbol{r}) \psi_{\alpha}(\boldsymbol{r}) .
$$

The disorder potential $U_{i m p}(\boldsymbol{r})$ is assumed to be a random function with zero mean and the correlator $\left\langle U_{i m p}\left(\boldsymbol{r}_{1}\right) U_{i m p}\left(\boldsymbol{r}_{2}\right)\right\rangle=n_{i m p} U_{0}^{2} \delta\left(\boldsymbol{r}_{1}-\boldsymbol{r}_{2}\right)$, where $n_{i m p}$ is the impurity concentration, and $U_{0}$ is the strength of an individual point-like impurity. The electron field operators have the form

$$
\psi_{\alpha}(\boldsymbol{r})=\frac{1}{\sqrt{\mathcal{V}}} \sum_{\boldsymbol{k}, \lambda} u_{\alpha \lambda}(\boldsymbol{k}) e^{i \boldsymbol{k} \boldsymbol{r}} c_{\boldsymbol{k} \lambda}
$$

from which we obtain the band representation of the impurity Hamiltonian:

$$
H_{i m p}=\frac{1}{\mathcal{V}} \sum_{\boldsymbol{k} \boldsymbol{k}^{\prime}} \sum_{\lambda \lambda^{\prime}} U_{i m p}\left(\boldsymbol{k}-\boldsymbol{k}^{\prime}\right) w_{\lambda \lambda^{\prime}}\left(\boldsymbol{k}, \boldsymbol{k}^{\prime}\right) c_{\boldsymbol{k} \lambda}^{\dagger} c_{\boldsymbol{k}^{\prime} \lambda^{\prime}}
$$

Here $U_{i m p}(\boldsymbol{q})$ is the Fourier transform of the impurity potential, $\left\langle U_{i m p}(\boldsymbol{q}) U_{i m p}\left(\boldsymbol{q}^{\prime}\right)\right\rangle=n_{i m p} U_{0}^{2} \mathcal{V} \delta_{\boldsymbol{q},-\boldsymbol{q}^{\prime}}$, and $\hat{w}\left(\boldsymbol{k}, \boldsymbol{k}^{\prime}\right)=\hat{u}^{\dagger}(\boldsymbol{k}) \hat{u}\left(\boldsymbol{k}^{\prime}\right)=\hat{w}^{\dagger}\left(\boldsymbol{k}^{\prime}, \boldsymbol{k}\right)$. We see that the impurity scattering amplitude in the band representation acquires both intraband and interband contributions and also becomes anisotropic, even for isotropic impurities. ${ }^{12}$

Averaging with respect to the impurity positions restores translational invariance: $\left\langle\mathcal{G}\left(\boldsymbol{k}_{1}, \boldsymbol{k}_{2} ; \omega_{n}\right)\right\rangle_{\text {imp }}=$ $\delta_{\boldsymbol{k}_{1}, \boldsymbol{k}_{2}} \mathcal{G}\left(\boldsymbol{k}, \omega_{n}\right)$. The disorder-averaged Green's function here has the same matrix structure as in the clean case, see Eq. (13). The magnetization in the superconducting state is determined by Eq. (14), in which the Green's function of the clean normal metal should be replaced by its disorder average.

The average matrix Green's function satisfies the Gor'kov equations: $\left(\mathcal{G}_{0}^{-1}-\Sigma_{i m p}-\Sigma_{Z}\right) \mathcal{G}=1$, where $\mathcal{G}_{0}$ 
is the Green's function at zero field in the absence of impurities, given by Eq. (15), the impurity self-energy in the self-consistent Born approximation is

$$
\begin{aligned}
& \Sigma_{i m p}\left(\boldsymbol{k}, \omega_{n}\right)=n_{i m p} U_{0}^{2} \\
& \quad \times \int \frac{d^{3} \boldsymbol{k}^{\prime}}{(2 \pi)^{3}} W\left(\boldsymbol{k}, \boldsymbol{k}^{\prime}\right) \mathcal{G}\left(\boldsymbol{k}^{\prime}, \omega_{n}\right) W\left(\boldsymbol{k}^{\prime}, \boldsymbol{k}\right),
\end{aligned}
$$

and $\Sigma_{Z}$ is the Zeeman self-energy (16). The $4 \times 4$ matrix $W$ is defined as follows:

$$
W\left(\boldsymbol{k}, \boldsymbol{k}^{\prime}\right)=\left(\begin{array}{cc}
\hat{w}\left(\boldsymbol{k}, \boldsymbol{k}^{\prime}\right) & 0 \\
0 & -\hat{w}^{T}\left(-\boldsymbol{k}^{\prime},-\boldsymbol{k}\right)
\end{array}\right) .
$$

It is straightforward to show that $\left[\hat{w}^{T}\left(-\boldsymbol{k}^{\prime},-\boldsymbol{k}\right)\right]_{\lambda \lambda^{\prime}}=$ $t_{\lambda}^{*}(\boldsymbol{k}) t_{\lambda^{\prime}}\left(\boldsymbol{k}^{\prime}\right) w_{\lambda \lambda^{\prime}}\left(\boldsymbol{k}, \boldsymbol{k}^{\prime}\right)$.

\section{A. Zero-field solution}

In the absence of magnetic field, $\Sigma_{Z}=0$, and, since $\mathcal{G}_{0}$ is band-diagonal, we seek the solution of the Gor'kov equation in a band-diagonal form given by Eqs. (18). For consistency, we require that the Nambu matrix components of the self-energy are also band-diagonal:

$$
\left(\Sigma_{i m p}\right)_{\lambda \lambda^{\prime}}^{a b}\left(\boldsymbol{k}, \omega_{n}\right)=\delta_{\lambda \lambda^{\prime}} \varrho_{\lambda}^{a b}(\boldsymbol{k}) \sigma_{\lambda}^{a b}\left(\boldsymbol{k}, \omega_{n}\right),
$$

where $a, b=1,2$ are the Nambu (particle-hole) indices, and $\varrho_{\lambda}^{11}(\boldsymbol{k})=\varrho_{\lambda}^{22}(\boldsymbol{k})=1, \varrho_{\lambda}^{12}(\boldsymbol{k})=t_{\lambda}(\boldsymbol{k}), \varrho_{\lambda}^{21}(\boldsymbol{k})=$ $t_{\lambda}^{*}(\boldsymbol{k})$. From Eq. (39) we obtain:

$$
\begin{aligned}
& \left(\begin{array}{ll}
\hat{\sigma}^{11}\left(\boldsymbol{k}, \omega_{n}\right) & \hat{\sigma}^{12}\left(\boldsymbol{k}, \omega_{n}\right) \\
\hat{\sigma}^{21}\left(\boldsymbol{k}, \omega_{n}\right) & \hat{\sigma}^{22}\left(\boldsymbol{k}, \omega_{n}\right)
\end{array}\right) \\
& =n_{i m p} U_{0}^{2} \int \frac{d^{3} \boldsymbol{k}^{\prime}}{(2 \pi)^{3}} \hat{w}\left(\boldsymbol{k}, \boldsymbol{k}^{\prime}\right) \\
& \times\left(\begin{array}{cc}
\hat{G}\left(\boldsymbol{k}^{\prime}, \omega_{n}\right) & \hat{\tilde{F}}\left(\boldsymbol{k}^{\prime}, \omega_{n}\right) \\
\hat{\tilde{F}}^{\dagger}\left(\boldsymbol{k}^{\prime}, \omega_{n}\right) & -\hat{G}^{T}\left(-\boldsymbol{k}^{\prime},-\omega_{n}\right)
\end{array}\right) \hat{w}\left(\boldsymbol{k}^{\prime}, \boldsymbol{k}\right) .
\end{aligned}
$$

Using the identity (33) one can show that the integrands on the right-hand side of these equations have the following form:

$$
\begin{aligned}
\hat{u}\left(\boldsymbol{k}^{\prime}\right) \hat{G}\left(\boldsymbol{k}^{\prime}, \omega_{n}\right) \hat{u}^{\dagger}\left(\boldsymbol{k}^{\prime}\right) \\
=\frac{G_{+}\left(\boldsymbol{k}^{\prime}, \omega_{n}\right)+G_{-}\left(\boldsymbol{k}^{\prime}, \omega_{n}\right)}{2} \hat{\tau}_{0} \\
\quad+\frac{G_{+}\left(\boldsymbol{k}^{\prime}, \omega_{n}\right)-G_{-}\left(\boldsymbol{k}^{\prime}, \omega_{n}\right)}{2} \hat{\boldsymbol{\gamma}}\left(\boldsymbol{k}^{\prime}\right) \hat{\boldsymbol{\tau}},
\end{aligned}
$$

etc. Assuming that $G_{\lambda}$ and $\tilde{F}_{\lambda}$ are even functions of momentum (the self-consistency of this will be verified below), the last line in Eq. (42) is odd in $\boldsymbol{k}^{\prime}$ and therefore vanishes after the $\boldsymbol{k}^{\prime}$-integration. Then,

$$
\begin{aligned}
& \sigma_{\lambda}^{11}\left(\boldsymbol{k}, \omega_{n}\right)=-\sigma_{\lambda}^{22}\left(\boldsymbol{k},-\omega_{n}\right)=\Sigma_{1}\left(\omega_{n}\right), \\
& \sigma_{\lambda}^{12}\left(\boldsymbol{k}, \omega_{n}\right)=\sigma_{\lambda}^{21, *}\left(\boldsymbol{k}, \omega_{n}\right)=\Sigma_{2}\left(\omega_{n}\right),
\end{aligned}
$$

where

$$
\begin{aligned}
& \Sigma_{1}\left(\omega_{n}\right)=\frac{1}{2} n_{i m p} U_{0}^{2} \sum_{\lambda} \int \frac{d^{3} \boldsymbol{k}}{(2 \pi)^{3}} G_{\lambda}\left(\boldsymbol{k}, \omega_{n}\right), \\
& \Sigma_{2}\left(\omega_{n}\right)=\frac{1}{2} n_{i m p} U_{0}^{2} \sum_{\lambda} \int \frac{d^{3} \boldsymbol{k}}{(2 \pi)^{3}} \tilde{F}_{\lambda}\left(\boldsymbol{k}, \omega_{n}\right) .
\end{aligned}
$$

Absorbing the real part of $\Sigma$ into the chemical potential, we have $\Sigma_{1}\left(\omega_{n}\right)=i \tilde{\Sigma}_{1}\left(\omega_{n}\right)$, where $\tilde{\Sigma}_{1}$ is odd in $\omega_{n}$.

Solving the Gor'kov equations we obtain the disorderaveraged Green's functions:

$$
\begin{aligned}
& G_{\lambda}\left(\boldsymbol{k}, \omega_{n}\right)=-\frac{i \tilde{\omega}_{n}+\xi_{\lambda}(\boldsymbol{k})}{\tilde{\omega}_{n}^{2}+\xi_{\lambda}^{2}(\boldsymbol{k})+\left|D_{\lambda}\left(\boldsymbol{k}, \omega_{n}\right)\right|^{2}}, \\
& \tilde{F}_{\lambda}\left(\boldsymbol{k}, \omega_{n}\right)=\frac{D_{\lambda}\left(\boldsymbol{k}, \omega_{n}\right)}{\tilde{\omega}_{n}^{2}+\xi_{\lambda}^{2}(\boldsymbol{k})+\left|D\left(\boldsymbol{k}, \omega_{n}\right)\right|^{2}},
\end{aligned}
$$

where $\tilde{\omega}_{n}=\omega_{n}-\tilde{\Sigma}_{1}\left(\omega_{n}\right)$ and $D_{\lambda}\left(\boldsymbol{k}, \omega_{n}\right)=\tilde{\Delta}_{\lambda}(\boldsymbol{k})+$ $\Sigma_{2}\left(\omega_{n}\right)$. Substituting these expressions into Eqs. (43), we arrive at the self-consistency equations for the renormalized Matsubara frequency and the gap functions:

$$
\begin{aligned}
\tilde{\omega}_{n}=\omega_{n} & +\frac{\Gamma}{2} \sum_{\lambda} \rho_{\lambda}\left\langle\frac{\tilde{\omega}_{n}}{\sqrt{\tilde{\omega}_{n}^{2}+\left|D_{\lambda}\left(\boldsymbol{k}, \omega_{n}\right)\right|^{2}}}\right\rangle_{\lambda}, \\
D_{\lambda}\left(\boldsymbol{k}, \omega_{n}\right) & =\tilde{\Delta}_{\lambda}(\boldsymbol{k}) \\
& +\frac{\Gamma}{2} \sum_{\lambda^{\prime}} \rho_{\lambda^{\prime}}\left\langle\frac{D_{\lambda^{\prime}}\left(\boldsymbol{k}, \omega_{n}\right)}{\sqrt{\tilde{\omega}_{n}^{2}+\mid D_{\lambda^{\prime}}\left(\boldsymbol{k}, \omega_{n}\right)^{2}}}\right\rangle_{\lambda^{\prime}} .
\end{aligned}
$$

Here $\Gamma=1 / 2 \tau$ is the elastic scattering rate, and $\tau=$ $\left(2 \pi n_{i m p} U_{0}^{2} N_{F}\right)^{-1}$ is the electron mean free time due to impurities. One can now see that, while $\tilde{\omega}_{n}$ is odd in $\omega_{n}$, $D_{\lambda}$ are even in both $\boldsymbol{k}$ and $\omega_{n}$, so that our assumptions are self-consistent.

\section{B. Spin susceptibility}

At $\boldsymbol{B} \neq 0$, both the Green's function and the impurity self-energy acquire field-dependent corrections: $\mathcal{G}_{\boldsymbol{B} \neq 0}=$ $\mathcal{G}+\delta \mathcal{G}, \Sigma_{i m p, \boldsymbol{B} \neq 0}=\Sigma_{i m p}+\delta \Sigma_{i m p}$. Here $\mathcal{G}$ and $\Sigma_{i m p}$ are the average Green's function and the impurity selfenergy at zero field found in Sec. IIIA Treating the Zeeman coupling as a small perturbation, we find $\delta \mathcal{G}=$ $\mathcal{G}\left(\Sigma_{Z}+\delta \Sigma_{i m p}\right) \mathcal{G}$.

Magnetization $M$ is given by Eq. (14). The contribution from the zero-field Green's function vanishes after the momentum integration, meaning that there is no spontaneous magnetism of the Cooper pairs in the superconducting state. Thus the magnetization is determined by $\delta \hat{G}$ and can be written as $\boldsymbol{M}=\boldsymbol{M}_{1}+\boldsymbol{M}_{2}$, where

$$
\boldsymbol{M}_{1}=T \sum_{n} \int \frac{d^{3} \boldsymbol{k}}{(2 \pi)^{3}} \operatorname{tr} \hat{\boldsymbol{m}}\left(\mathcal{G} \Sigma_{Z} \mathcal{G}\right)^{11}
$$


corresponds diagrammatically to a "bubble" containing two disorder-averaged Green's functions, while

$$
\boldsymbol{M}_{2}=T \sum_{n} \int \frac{d^{3} \boldsymbol{k}}{(2 \pi)^{3}} \operatorname{tr} \hat{\boldsymbol{m}}\left(\mathcal{G} \delta \Sigma_{i m p} \mathcal{G}\right)^{11}
$$

describes the impurity vertex corrections (recall that the upper indices here label the Nambu matrix components).

The contribution to the susceptibility from $\boldsymbol{M}_{1}$ can be calculated similarly to the clean case in Sec. II $\chi_{1, i j}=\partial M_{1, i} / \partial B_{j}=\chi_{i j}^{+}+\chi_{i j}^{-}+\tilde{\chi}_{i j}$, where $\chi_{i j}^{\lambda}$ are the intraband and $\tilde{\chi}_{i j}$ the interband susceptibilities. Since the latter is determined by all quasiparticles between the "+" and "-" Fermi surfaces, its change below the superconducting transition is negligibly small. One can show that the interband susceptibility in the normal state is not sensitive to impurities, therefore $\tilde{\chi}_{i j}$ is given by its clean normal state expression (25). The intraband susceptibilities are given by

$$
\begin{aligned}
\chi_{i j}^{\lambda}= & -\mu_{B}^{2} T \sum_{n} \int \frac{d^{3} \boldsymbol{k}}{(2 \pi)^{3}} \hat{\gamma}_{i} \hat{\gamma}_{j}\left(G_{\lambda}^{2}+\left|\tilde{F}_{\lambda}\right|^{2}\right) \\
= & \mu_{B}^{2} N_{\lambda}\left\langle\hat{\gamma}_{i} \hat{\gamma}_{j}\right\rangle_{\lambda} \\
& -\pi \mu_{B}^{2} N_{\lambda} T \sum_{n}\left\langle\hat{\gamma}_{i} \hat{\gamma}_{j} \frac{\left|D_{\lambda}\right|^{2}}{\left(\tilde{\omega}_{n}^{2}+\left|D_{\lambda}\right|^{2}\right)^{3 / 2}}\right\rangle_{\lambda},
\end{aligned}
$$

The Green's functions in the first line here are given by the disorder-averaged expressions (44). In contrast to the clean case, however, it is not possible to calculate the Matsubara sums before the momentum integrals. In order to do the momentum integrals first, one should ${ }^{29}$ add and subtract the normal-state intraband susceptibility, which is not affected by impurities, see Eq. (27).

Let us now calculate the impurity vertex corrections (48). Substituting $\delta \mathcal{G}=\mathcal{G}\left(\Sigma_{Z}+\delta \Sigma_{i m p}\right) \mathcal{G}$ into Eq. (39), we obtain the following equation for $\delta \Sigma_{i m p}$ :

$$
\begin{array}{r}
\delta \Sigma_{i m p}\left(\boldsymbol{k}, \omega_{n}\right)-n_{i m p} U_{0}^{2} \int \frac{d^{3} \boldsymbol{k}^{\prime}}{(2 \pi)^{3}} W\left(\boldsymbol{k}, \boldsymbol{k}^{\prime}\right) \mathcal{G}\left(\boldsymbol{k}^{\prime}, \omega_{n}\right) \\
\times \delta \Sigma_{i m p}\left(\boldsymbol{k}^{\prime}, \omega_{n}\right) \mathcal{G}\left(\boldsymbol{k}^{\prime}, \omega_{n}\right) W\left(\boldsymbol{k}^{\prime}, \boldsymbol{k}\right) \\
=n_{i m p} U_{0}^{2} \int \frac{d^{3} \boldsymbol{k}^{\prime}}{(2 \pi)^{3}} W\left(\boldsymbol{k}, \boldsymbol{k}^{\prime}\right) \mathcal{G}\left(\boldsymbol{k}^{\prime}, \omega_{n}\right) \\
\times \Sigma_{Z}\left(\boldsymbol{k}^{\prime}\right) \mathcal{G}\left(\boldsymbol{k}^{\prime}, \omega_{n}\right) W\left(\boldsymbol{k}^{\prime}, \boldsymbol{k}\right) .
\end{array}
$$

The contributions to $\boldsymbol{M}_{2}$ from the interband components of $\delta \Sigma_{i m p}$ can be neglected compared to those from the intraband components, because the former contain the momentum integrals of the products of the Green's functions from different bands, which are at least by a factor of $|\tilde{\Delta}| /|\gamma| \ll 1$ smaller than their same-band counterparts. One can seek the field-induced correction to the impurity self-energy in the band-diagonal form similar to Eq. (40):

$$
\left(\delta \Sigma_{i m p}\right)_{\lambda \lambda^{\prime}}^{a b}\left(\boldsymbol{k}, \omega_{n}\right)=\delta_{\lambda \lambda^{\prime}} \varrho_{\lambda}^{a b}(\boldsymbol{k}) \delta \sigma_{\lambda}^{a b}\left(\boldsymbol{k}, \omega_{n}\right) .
$$

For the same reason, one should retain only those terms on the right-hand side of Eq. (50) in which both
Green's functions have the same band index. Since $\boldsymbol{m}_{\lambda \lambda}(-\boldsymbol{k})=-\boldsymbol{m}_{\lambda \lambda}(\boldsymbol{k})=-\lambda \mu_{B} \hat{\gamma}(\boldsymbol{k})$, see Eqs. (8), we have $\left(\Sigma_{Z}\right)_{\lambda \lambda}^{11}(\boldsymbol{k})=\left(\Sigma_{Z}\right)_{\lambda \lambda}^{22}(\boldsymbol{k})=-\lambda \mu_{B} \hat{\gamma}(\boldsymbol{k})$. Inserting this and the expressions (51) in Eq. (50) and using the identity

$$
\left|w_{\lambda \lambda^{\prime}}\left(\boldsymbol{k}, \boldsymbol{k}^{\prime}\right)\right|^{2}=\frac{1+\lambda \lambda^{\prime} \hat{\gamma}(\boldsymbol{k}) \hat{\gamma}\left(\boldsymbol{k}^{\prime}\right)}{2}
$$

we obtain the Nambu matrix components of the righthand side of Eq. (50):

$$
\begin{array}{r}
(R . H . S .)_{\lambda}^{a b}\left(\boldsymbol{k}, \omega_{n}\right)=-\lambda \mu_{B} \varrho_{\lambda}^{a b}(\boldsymbol{k}) \sum_{i j} \hat{\gamma}_{i}(\boldsymbol{k}) B_{j} \\
\times \frac{1}{2} n_{i m p} U_{0}^{2} \sum_{\lambda^{\prime}} \int \frac{d^{3} \boldsymbol{k}^{\prime}}{(2 \pi)^{3}} \hat{\gamma}_{i} \hat{\gamma}_{j} R_{\lambda^{\prime}}^{a b}
\end{array}
$$

where $R_{\lambda}^{11}=G_{\lambda}^{2}+\left|\tilde{F}_{\lambda}\right|^{2}, R_{\lambda}^{12}=\left(G_{\lambda}-\bar{G}_{\lambda}\right) \tilde{F}_{\lambda}, R_{\lambda}^{21}=$ $\left(G_{\lambda}-\bar{G}_{\lambda}\right) \tilde{F}_{\lambda}^{*}, R_{\lambda}^{22}=\bar{G}_{\lambda}^{2}+\left|\tilde{F}_{\lambda}\right|^{2}$, and $\bar{G}_{\lambda}\left(\boldsymbol{k}, \omega_{n}\right)=$ $G_{\lambda}\left(-\boldsymbol{k},-\omega_{n}\right)$. This suggests that one can seek $\delta \sigma_{\lambda}^{a b}$ in the following form:

$$
\begin{aligned}
& \delta \sigma_{\lambda}^{11}\left(\boldsymbol{k}, \omega_{n}\right)=\delta \sigma_{\lambda}^{22}\left(\boldsymbol{k}, \omega_{n}\right)=\lambda \hat{\gamma}(\boldsymbol{k}) \boldsymbol{X}\left(\omega_{n}\right), \\
& \delta \sigma_{\lambda}^{12}\left(\boldsymbol{k}, \omega_{n}\right)=-\delta \sigma_{\lambda}^{21, *}\left(\boldsymbol{k}, \omega_{n}\right)=\lambda \hat{\gamma}(\boldsymbol{k}) \boldsymbol{Y}\left(\omega_{n}\right) .
\end{aligned}
$$

After some straightforward algebra, we obtain from Eq. (50) the linear equations for $\boldsymbol{X}\left(\omega_{n}\right)$ and $\boldsymbol{Y}\left(\omega_{n}\right)$ :

$$
\begin{aligned}
& X_{i}-\sum_{j}\left(A_{1, i j} X_{j}+A_{2, i j} Y_{j}+A_{2, i j}^{*} Y_{j}^{*}\right)=X_{0, i} \\
& Y_{i}-\sum_{j}\left(2 A_{2, i j}^{*} X_{j}+A_{3, i j} Y_{j}+A_{4, i j} Y_{j}^{*}\right)=Y_{0, i} .
\end{aligned}
$$

The notations here are as follows:

$$
\begin{aligned}
& A_{1, i j}\left(\omega_{n}\right)=\frac{\Gamma}{2} \sum_{\lambda} \rho_{\lambda}\left\langle\hat{\gamma}_{i} \hat{\gamma}_{j} \frac{\left|D_{\lambda}\right|^{2}}{\left(\tilde{\omega}_{n}^{2}+\left|D_{\lambda}\right|^{2}\right)^{3 / 2}}\right\rangle_{\lambda}, \\
& A_{2, i j}\left(\omega_{n}\right)=\frac{\Gamma}{4} \sum_{\lambda} \rho_{\lambda}\left\langle\hat{\gamma}_{i} \hat{\gamma}_{j} \frac{i \tilde{\omega}_{n} D_{\lambda}^{*}}{\left(\tilde{\omega}_{n}^{2}+\left|D_{\lambda}\right|^{2}\right)^{3 / 2}}\right\rangle_{\lambda} \\
& A_{3, i j}\left(\omega_{n}\right)=\frac{\Gamma}{4} \sum_{\lambda} \rho_{\lambda}\left\langle\hat{\gamma}_{i} \hat{\gamma}_{j} \frac{2 \tilde{\omega}_{n}^{2}+\left|D_{\lambda}\right|^{2}}{\left(\tilde{\omega}_{n}^{2}+\left|D_{\lambda}\right|^{2}\right)^{3 / 2}}\right\rangle_{\lambda}, \\
& A_{4, i j}\left(\omega_{n}\right)=\frac{\Gamma}{4} \sum_{\lambda} \rho_{\lambda}\left\langle\hat{\gamma}_{i} \hat{\gamma}_{j} \frac{D_{\lambda}^{2}}{\left(\tilde{\omega}_{n}^{2}+\left|D_{\lambda}\right|^{2}\right)^{3 / 2}}\right\rangle_{\lambda} \\
& X_{0, i}\left(\omega_{n}\right)=-\mu_{B} \sum_{j} A_{1, i j}\left(\omega_{n}\right) B_{j}, \\
& Y_{0, i}\left(\omega_{n}\right)=-2 \mu_{B} \sum_{j} A_{2, i j}^{*}\left(\omega_{n}\right) B_{j} .
\end{aligned}
$$

We see that, while $\boldsymbol{X}\left(\omega_{n}\right)$ is real, $\boldsymbol{Y}\left(\omega_{n}\right)$ is complex, and both are linear functions of $\boldsymbol{B}$. For arbitrary complex gap functions $\tilde{\Delta}_{\lambda}(\boldsymbol{k})$, Eqs. (53) can be transformed into a system of nine equations for the components of $\boldsymbol{X}\left(\omega_{n}\right)$, $\operatorname{Re} \boldsymbol{Y}\left(\omega_{n}\right)$, and $\operatorname{Im} \boldsymbol{Y}\left(\omega_{n}\right)$. 
Plugging the expressions (51) and (52) into Eq. (48), we obtain:

$$
\begin{array}{r}
M_{2, i}=\mu_{B} T \sum_{n} \sum_{\lambda} \int \frac{d^{3} \boldsymbol{k}}{(2 \pi)^{3}} \hat{\gamma}_{i} \hat{\gamma}_{j}\left[\left(G_{\lambda}^{2}+\left|\tilde{F}_{\lambda}\right|^{2}\right) X_{j}\right. \\
\left.-\left(G_{\lambda} \tilde{F}_{\lambda}^{*}\right) Y_{j}+\left(G_{\lambda} \tilde{F}_{\lambda}\right) Y_{j}^{*}\right] \\
=4 \pi \mu_{B} N_{F} \tau T \sum_{n}\left[X_{i}\left(\omega_{n}\right)-X_{0, i}\left(\omega_{n}\right)\right]
\end{array}
$$

[here we also used the first of the equations (53)]. The vertex correction to the susceptibility is then found from $\chi_{2, i j}=\partial M_{2, i} / \partial B_{j}$.

The total spin susceptibility has the form $\chi_{i j}=\chi_{1, i j}+$ $\chi_{2, i j}=\tilde{\chi}_{i j}+\chi_{i j}^{+}+\chi_{i j}^{-}+\chi_{2, i j}$. Putting together Eqs. (49) and (54), we arrive at the following final expression:

$$
\chi_{i j}=\chi_{N, i j}+4 \pi \mu_{B} N_{F} \tau T \sum_{n} \frac{\partial X_{i}\left(\omega_{n}\right)}{\partial B_{j}},
$$

where $\chi_{N, i j}$ is the normal-state susceptibility (27), which is not sensitive to disorder. At $\Gamma \rightarrow 0$, we have $\boldsymbol{X}\left(\omega_{n}\right) \rightarrow$ $\boldsymbol{X}_{0}\left(\omega_{n}\right)$, with $\tilde{\omega}_{n}=\omega_{n}$ and $D_{\lambda}=\tilde{\Delta}_{\lambda}$. Using the identity

$$
\pi T \sum_{n} \frac{\left|\tilde{\Delta}_{\lambda}(\boldsymbol{k})\right|^{2}}{\left(\omega_{n}^{2}+\left|\tilde{\Delta}_{\lambda}(\boldsymbol{k})\right|^{2}\right)^{3 / 2}}=1-Y_{\lambda}(\boldsymbol{k}, T),
$$

where $Y_{\lambda}$ is given by Eq. (23), we recover the susceptibility (26) of a clean superconductor.

\section{RESIDUAL SUSCEPTIBILITY}

The equations derived in the previous section cannot be solved analytically in the general case. On the other hand, application of our results to real noncentrosymmetric materials is complicated by the lack of a definite information about the superconducting gap symmetry and the distribution of the pairing strength between the bands. Therefore one can only make progress by using some simple models.

First, we assume that the pairing corresponds to the unity representation of the point group and is fully isotropic: $\phi_{+}(\boldsymbol{k})=\phi_{-}(\boldsymbol{k})=1$, i.e. $\tilde{\Delta}_{\lambda}(\boldsymbol{k})=\eta_{\lambda}$. Then it follows from Eqs. (46) that $D_{\lambda}\left(\boldsymbol{k}, \omega_{n}\right)=D\left(\omega_{n}\right)$ and the order parameter components (which can be chosen to be real) satisfy the self-consistency equations:

$$
\begin{array}{r}
\sum_{\lambda^{\prime}} V_{\lambda \lambda^{\prime}}^{-1} \eta_{\lambda^{\prime}}=T \sum_{n} \int \frac{d^{3} \boldsymbol{k}}{(2 \pi)^{3}} \tilde{F}_{\lambda}\left(\boldsymbol{k}, \omega_{n}\right) \\
=\pi \rho_{\lambda} N_{F} T \sum_{n} \frac{D_{\lambda}\left(\omega_{n}\right)}{\sqrt{\tilde{\omega}_{n}^{2}+D_{\lambda}^{2}\left(\omega_{n}\right)}},
\end{array}
$$

where $\tilde{\omega}_{n}$ is found from Eq. (45). The Matsubara sum is cut off at the limiting frequency of the order of the BCS shell width $\varepsilon_{c}$.

We further assume that the SO coupling is weak compared to the Fermi energy, $|\gamma| / \epsilon_{F} \ll 1$, and that the pairing strength, see Eq. (10) does not vary between the bands: $V_{++}=V_{--}>0$. Since the matrix of the coupling constants is symmetric and positive-definite, we also have $V_{+-}=V_{-+}$and $V_{++}>\left|V_{+-}\right|$. The selfconsistency equations have two solutions: 1) the order parameter magnitudes and phases in the two bands are the same: $\eta_{+}=\eta_{-}=\eta$, and 2) the magnitudes are the same, but the signs are opposite: $\eta_{+}=-\eta_{-}=\eta$. According to Sec. IIB, the former solution corresponds to the singlet state in the spin representation, while the latter - to the "protected" triplet state. While in the clean limit the spin susceptibility for both states is given by Eq. (29), the effects of impurities in the two cases have to be analyzed separately.

$$
\text { A. } \eta_{+}=\eta_{-}=\eta
$$

In this case $D_{+}=D_{-}=D\left(\omega_{n}\right)$, and Eqs. (45) and (46) take the following form:

$$
\begin{aligned}
& D=\eta+\Gamma \frac{D}{\sqrt{\tilde{\omega}_{n}^{2}+D^{2}}}, \\
& \tilde{\omega}_{n}=\omega_{n}+\Gamma \frac{\tilde{\omega}_{n}}{\sqrt{\tilde{\omega}_{n}^{2}+D^{2}}} .
\end{aligned}
$$

The solution of these equations is $D\left(\omega_{n}\right)=Z\left(\omega_{n}\right) \eta, \tilde{\omega}_{n}=$ $Z\left(\omega_{n}\right) \omega_{n}$, where $Z\left(\omega_{n}\right)=1+\Gamma / \sqrt{\omega_{n}^{2}+\eta^{2}}$. The gap equation (56) becomes

$$
\eta=\pi g_{1} T \sum_{n} \frac{D}{\sqrt{\tilde{\omega}_{n}^{2}+D^{2}}}=\pi g_{1} T \sum_{n} \frac{\eta}{\sqrt{\omega_{n}^{2}+\eta^{2}}},
$$

where $g_{1}=\left(V_{++}+V_{+-}\right) N_{F}$ is the dimensionless coupling constant. Note that the scattering rate has dropped out of the gap equation, so that there is an analog of the Anderson theorem: neither the gap magnitude nor the critical temperature are affected by impurities: $T_{c}(\Gamma)=$ $T_{c 1}=\left(2 \gamma \epsilon_{c} / \pi\right) e^{-1 / g_{1}}(\ln \gamma \simeq 0.577$ is Euler's constant $)$. In particular, the gap magnitude at $T=0$ is given by the clean BCS expression: $\eta(T=0)=\eta_{0}=(\pi / \gamma) T_{c 1}$.

Let us now calculate the spin susceptibility, using Eq. (55). Since the gap functions are real, $\boldsymbol{Y}$ is purely imaginary: $\boldsymbol{Y}\left(\omega_{n}\right)=i \tilde{\boldsymbol{Y}}\left(\omega_{n}\right)$, and Eqs. (53) take the following form:

$$
\left(\begin{array}{cc}
1-\hat{C}_{1} & \hat{C}_{2} \\
\hat{C}_{2} & 1-\hat{C}_{3}
\end{array}\right)\left(\begin{array}{c}
\boldsymbol{X} \\
\tilde{\boldsymbol{Y}}
\end{array}\right)=\left(\begin{array}{c}
-\mu_{B} \hat{C}_{1} \boldsymbol{B} \\
\mu_{B} \hat{C}_{2} \boldsymbol{B}
\end{array}\right),
$$

where $C_{m, i j}=C_{m}\left\langle\hat{\gamma}_{i} \hat{\gamma}_{j}\right\rangle_{F}, m=1,2,3$, and

$$
\begin{aligned}
& C_{1}\left(\omega_{n}\right)=\Gamma \frac{1}{Z\left(\omega_{n}\right)} \frac{\eta^{2}}{\left(\omega_{n}^{2}+\eta^{2}\right)^{3 / 2}}, \\
& C_{2}\left(\omega_{n}\right)=\Gamma \frac{1}{Z\left(\omega_{n}\right)} \frac{\omega_{n} \eta}{\left(\omega_{n}^{2}+\eta^{2}\right)^{3 / 2}}, \\
& C_{3}\left(\omega_{n}\right)=\Gamma \frac{1}{Z\left(\omega_{n}\right)} \frac{\omega_{n}^{2}}{\left(\omega_{n}^{2}+\eta^{2}\right)^{3 / 2}} .
\end{aligned}
$$




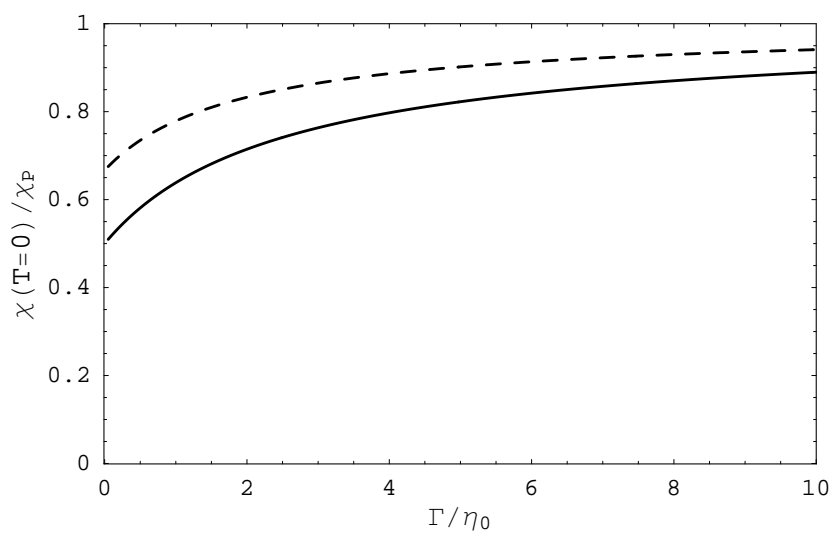

FIG. 2: The residual susceptibility vs disorder strength for $\eta_{+}=\eta_{-}=\eta_{0}$. The solid line corresponds to the transverse components in the $2 \mathrm{D}$ case $\left(\chi_{z z}=\chi_{P}\right.$ and is disorderindependent), the dashed line - to all three diagonal components in the $3 \mathrm{D}$ case.

Substituting the solution of the equations (58) in Eq. (55), we obtain:

$$
\chi_{i j}=\chi_{P} \delta_{i j}-\pi \mu_{B}^{2} N_{F} T \sum_{n} \Lambda_{i j}\left(\omega_{n}\right),
$$

where $\hat{\Lambda}=4 \tau(1-\hat{L})^{-1} \hat{L}$, and $\hat{L}=\hat{C}_{1}+\hat{C}_{2}\left(1-\hat{C}_{3}\right)^{-1} \hat{C}_{2}$. In the coordinate system in which $\left\langle\hat{\gamma}_{i} \hat{\gamma}_{j}\right\rangle_{F}$ is diagonal, the susceptibility tensor is also diagonal:

$$
\frac{\chi_{i i}(T)}{\chi_{P}}=1-\left\langle\hat{\gamma}_{i}^{2}\right\rangle_{F} \pi T \sum_{n} \frac{\eta^{2}}{\omega_{n}^{2}+\eta^{2}} \frac{1}{\sqrt{\omega_{n}^{2}+\eta^{2}}+\Gamma_{i}}
$$

where $\Gamma_{i}=\left(1-\left\langle\hat{\gamma}_{i}^{2}\right\rangle_{F}\right) \Gamma$.

We are particularly interested in the effect of disorder on the residual susceptibility at $T=0$. In this limit, the Matsubara sum in the last formula can be replaced by a frequency integral, which gives

$$
\frac{\chi_{i i}(T=0)}{\chi_{P}}=1-\left\langle\hat{\gamma}_{i}^{2}\right\rangle_{F}+\left\langle\hat{\gamma}_{i}^{2}\right\rangle_{F} \Phi_{1}\left(\frac{\Gamma_{i}}{\eta_{0}}\right)
$$

where

$$
\Phi_{1}(x)=1-\frac{\pi}{2 x}\left(1-\frac{4}{\pi \sqrt{1-x^{2}}} \arctan \sqrt{\frac{1-x}{1+x}}\right)
$$

[at $x>1$ this function is evaluated using $\arctan (i x)=$ $\left.i \tanh ^{-1}(x)\right]$. While the first two terms on the right-hand side of the expression (61) represent the residual susceptibility in the clean case, see Eq. (28), the last term describes the impurity effect. In a weakly-disordered superconductor, using the asymptotics $\Phi_{1}(x) \simeq \pi x / 4$, we find that the residual susceptibility increases linearly with disorder. In the dirty limit, $\Gamma \gg \eta_{0}$, we have $\Phi_{1}(x) \rightarrow 1$, therefore $\chi_{i i}(T=0)$ approaches the normal-state value $\chi_{P}$. For the two simple band-structure models discussed in the end of Sec. IA the Fermi-surface averages can be calculated analytically, and we obtain the results plotted in Fig. 2,

Thus we see that, similarly to spin-orbit impurities in a usual centrosymmetric superconductor $\stackrel{26}{\underline{n}}$ scalar impurities in a noncentrosymmetric superconductor lead to an enhancement of the spin susceptibility at $T=0$. Since the interband contribution is not sensitive to disorder, this effect can be attributed to an increase in the intraband susceptibilities.

$$
\text { B. } \eta_{+}=-\eta_{-}=\eta
$$

In this case $D_{+}=-D_{-}=\eta$, and we obtain from Eqs. (45) and (56):

$$
\begin{aligned}
& \tilde{\omega}_{n}=\omega_{n}+\Gamma \frac{\tilde{\omega}_{n}}{\sqrt{\tilde{\omega}_{n}^{2}+\eta^{2}}}, \\
& \eta=\pi g_{2} T \sum_{n} \frac{\eta}{\sqrt{\tilde{\omega}_{n}^{2}+\eta^{2}}},
\end{aligned}
$$

where $g_{2}=\left(V_{++}-V_{+-}\right) N_{F}$. In the absence of impurities, the critical temperature is given by the BCS expression: $T_{c}(\Gamma=0)=T_{c 2}=\left(2 \gamma \epsilon_{c} / \pi\right) e^{-1 / g_{2}}$. If $V_{+-}>0$ (attractive interband interaction), then $g_{2}<g_{1}$ and $T_{c 2}<T_{c 1}$, i.e. the phase transition occurs into the state $\eta_{+}=\eta_{-}$. If $V_{+-}<0$ (repulsive interband interaction), then $g_{2}>g_{1}$ and $T_{c 2}>T_{c 1}$, i.e. the phase transition occurs into the state $\eta_{+}=-\eta_{-}$.

In contrast to the previous case, both the critical temperature and the gap magnitude are now suppressed by disorder. Indeed, linearizing the equations (63) and (64) with respect to $\eta$, we obtain the following equation for the critical temperature $T_{c}(\Gamma)$ :

$$
\ln \frac{T_{c 2}}{T_{c}}=\Psi\left(\frac{1}{2}+\frac{\Gamma}{2 \pi T_{c}}\right)-\Psi\left(\frac{1}{2}\right) .
$$

Thus the suppression of $T_{c}$ by scalar impurities is described by the same Abrikosov-Gor'kov function as in a conventional BCS superconductor with magnetic impurities. The superconductivity is completely destroyed if the disorder strength exceeds the critical value $\Gamma_{c}=$ $(\pi / 2 \gamma) T_{c 2}$.

To find the gap magnitude at $T=0$ we follow the procedure outlined in Ref. 31. Replacing the Matsubara sum by a frequency integral in the gap equation (64), we have

$$
\begin{array}{r}
\frac{1}{g_{2}}=\int_{0}^{\infty} d \omega\left(\frac{1}{\sqrt{\tilde{\omega}^{2}+\eta^{2}}}-\frac{1}{\sqrt{\omega^{2}+\eta^{2}}}\right) \\
+\int_{0}^{\varepsilon_{c}} d \omega \frac{1}{\sqrt{\omega^{2}+\eta^{2}}},
\end{array}
$$

where the function $\tilde{\omega}(\omega)$ is obtained from the continuous limit of Eq. [63):

$$
\tilde{\omega}=\omega+\Gamma \frac{\tilde{\omega}}{\sqrt{\tilde{\omega}^{2}+\eta^{2}}} .
$$


Since $\varepsilon_{c} \gg \eta$, the last integral in Eq. (66) is equal to $\ln \left(2 \varepsilon_{c} / \eta\right)$. In the clean case we recover the BCS expression for the gap magnitude at $T=0: \eta_{0}=(\pi / \gamma) T_{c 2}=$ $2 \Gamma_{c}$. In the presence of disorder Eq. (66) can be represented in the form

$$
\ln \frac{\eta_{0}}{\eta}=\int_{0}^{\infty} d \omega\left(\frac{1}{\sqrt{\omega^{2}+\eta^{2}}}-\frac{1}{\sqrt{\tilde{\omega}^{2}+\eta^{2}}}\right) .
$$

Using Eq. (67) the second term can be transformed into an integral over $\tilde{\omega}$. Introducing the notation $x=\Gamma / \eta$, we arrive at the following equation for the gap magnitude at zero temperature as a function of $\Gamma$ :

$$
\mathcal{F}(x)=\ln \frac{\Gamma_{c}}{\Gamma},
$$

where

$$
\begin{array}{r}
\mathcal{F}(x)=\frac{\pi x}{4}-\ln (2 x)+\theta(x-1)\left[\ln \left(x+\sqrt{x^{2}-1}\right)\right. \\
\left.-\frac{x}{2} \arctan \sqrt{x^{2}-1}-\frac{\sqrt{x^{2}-1}}{2 x}\right] .
\end{array}
$$

The equation (69) does not have solutions at $\Gamma>\Gamma_{c}$, which is consistent with the complete suppression of superconductivity above the critical disorder strength.

Repeating the steps from the previous subsection, we obtain the susceptibility in the form (59), where

$$
L_{i j}\left(\omega_{n}\right)=\Gamma \frac{\eta^{2}}{\left(\tilde{\omega}_{n}^{2}+\eta^{2}\right)^{3 / 2}}\left\langle\hat{\gamma}_{i} \hat{\gamma}_{j}\right\rangle_{F}
$$

In the coordinate system in which $\left\langle\hat{\gamma}_{i} \hat{\gamma}_{j}\right\rangle_{F}$ is diagonal, the nonzero components of the susceptibility tensor are given by

$$
\frac{\chi_{i i}(T)}{\chi_{P}}=1-\left\langle\hat{\gamma}_{i}^{2}\right\rangle_{F} \pi T \sum_{n} \frac{\eta^{2}}{\left(\tilde{\omega}_{n}^{2}+\eta^{2}\right)^{3 / 2}-\Gamma\left\langle\hat{\gamma}_{i}^{2}\right\rangle_{F} \eta^{2}} .
$$

We note that for a spherical 3D model with $\left\langle\hat{\gamma}_{i}^{2}\right\rangle_{F}=1 / 3$ this expression has exactly the same form as the susceptibility of the superfluid ${ }^{3} \mathrm{He}-\mathrm{B}$ in aerogel, see Refs. 31 and 32 .

At zero temperature we replace the Matsubara sum by a frequency integral and then transform it into an integral over $\tilde{\omega}$ using Eq. (67), with the following result:

$$
\frac{\chi_{i i}(T=0)}{\chi_{P}}=1-\left\langle\hat{\gamma}_{i}^{2}\right\rangle_{F}+\left\langle\hat{\gamma}_{i}^{2}\right\rangle_{F} \Phi_{2}\left(\frac{\Gamma}{\eta}\right)
$$

where

$$
\begin{array}{r}
\Phi_{2}(x)=1-\int_{y_{\text {min }}}^{\infty} d y\left[1-\frac{x}{\left(y^{2}+1\right)^{3 / 2}}\right] \\
\times \frac{1}{\left(y^{2}+1\right)^{3 / 2}-x\left\langle\hat{\gamma}_{i}^{2}\right\rangle_{F}},
\end{array}
$$

and $y_{\min }=\theta(x-1) \sqrt{x^{2}-1}$. The last term on the righthand side of Eq. (71) describes the effect of impurities

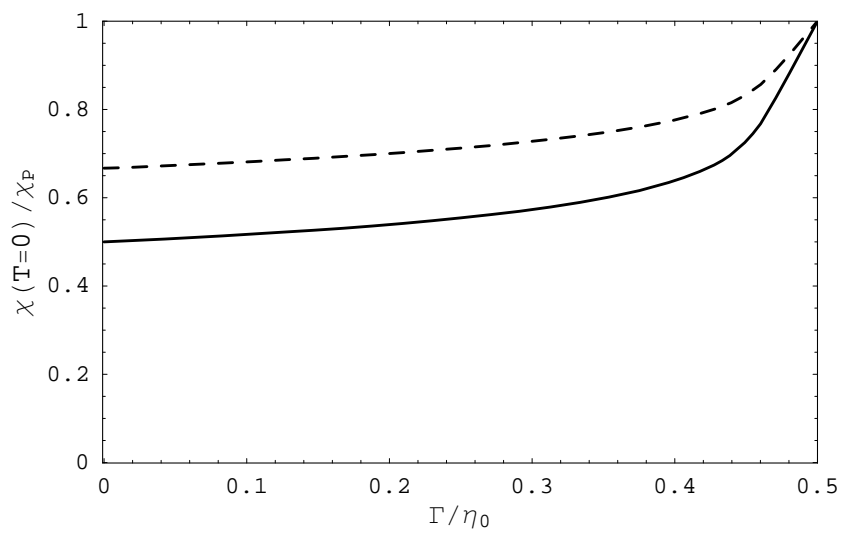

FIG. 3: The residual susceptibility vs disorder strength for $\eta_{+}=-\eta_{-}\left(\Gamma / \eta_{0}=0.5\right.$ corresponds to the critical disorder strength $\left.\Gamma=\Gamma_{c}\right)$. The solid line corresponds to the transverse components in the $2 \mathrm{D}$ case $\left(\chi_{z z}=\chi_{P}\right.$ and is disorderindependent), the dashed line - to all three diagonal components in the $3 \mathrm{D}$ case.

on the residual susceptibility. For each disorder strength, one first finds the solution $x(\Gamma)$ of Eq. (69) and then calculates $\Phi_{2}(x(\Gamma))$. In the weak disorder limit we have $x(\Gamma) \simeq \Gamma / 2 \Gamma_{c} \ll 1$, and $\Phi_{2}(x) \simeq(3 \pi x / 16)\left(1-\left\langle\hat{\gamma}_{i}^{2}\right\rangle_{F}\right)$, i.e. the residual susceptibility increases linearly with disorder. At $\Gamma \rightarrow \Gamma_{c}=\eta_{0} / 2: x(\Gamma) \simeq \sqrt{\Gamma_{c} / 12\left(\Gamma_{c}-\Gamma\right)} \gg 1$. In this limit $\Phi_{2}(x) \rightarrow 1$ and $\chi_{i i}(T=0) \rightarrow \chi_{P}$.

The dependence of $\chi_{i i}(T=0)$ on the disorder strength for the 2D and 3D models is plotted in Fig. 3. As in the case $\eta_{+}=\eta_{-}$, the residual susceptibility is enhanced by impurities.

\section{CONCLUSIONS}

The spin susceptibility of a clean noncentrosymmetric superconductor has a large residual value at $T=0$, which can be attributed to the temperature-independent contribution of the virtual transitions between the nondegenerate bands split by the SO coupling. We have studied the effects of disorder and found that scalar impurities in noncentrosymmetric superconductors act like spin-orbit impurities in centrosymmetric BCS superconductors, in the sense that they considerably enhance the residual susceptibility. The quantitative details depend on many factors, in particular the shape of the Fermi surface, the structure of the SO coupling, characterized by $\gamma(\boldsymbol{k})$, and the symmetry of the order parameter.

In the model in which the gap functions in both SO split bands are the same and isotropic, the critical temperature $T_{c}$ does not depend on the elastic scattering rate $\Gamma$, while the residual susceptibility increases and approaches the normal-state value $\chi_{P}$ in the dirty limit $\Gamma \rightarrow \infty$. If the two gap functions have the same magnitudes but opposite signs, $T_{c}$ is completely suppressed above the critical concentration of impurities corresponding to $\Gamma_{c}$, with the residual susceptibility approaching $\chi_{P}$ 
at $\Gamma \rightarrow \Gamma_{c}$.

The band structure models considered here cannot be directly applied to $\mathrm{CePt}_{3} \mathrm{Si}$, whose Fermi surface is quite complicated and consists of multiple sheets. ${ }^{7}$ It is not known which one (or ones) of them are superconducting. The order parameter symmetry is not known either, although there is strong experimental evidence that the superconducting order parameter has lines of gap nodes $33,34,35,36,37$ and likely corresponds to one of the nontrivial one-dimensional representations of the point group $\mathbf{C}_{4 v}$. We expect however that the qualitative picture described above remains valid for anisotropic pairing as well, namely the residual susceptibility increases in the presence of impurities.

\section{Acknowledgments}

The author is pleased to thank V. P. Mineev for stimulating discussions. The financial support from the Natural Sciences and Engineering Research Council of Canada and the Brock University Research Excellence Chair Program is gratefully acknowledged.

\section{APPENDIX A: SPIN-ORBIT COUPLING OF BAND ELECTRONS}

In order to derive the expression (1), we start with the Hamiltonian for non-interacting electrons in a perfect crystal lattice:

$$
H_{0}=-\frac{\hbar^{2}}{2 m} \nabla^{2}+U(\boldsymbol{r})-i \frac{\hbar^{2}}{4 m^{2} c^{2}} \hat{\boldsymbol{\sigma}}[\boldsymbol{\nabla} U(\boldsymbol{r}) \times \nabla],
$$

where $U(\boldsymbol{r})$ is the lattice potential. The last term, $H_{S O}$, represents the spin-orbit coupling. In the absence of the latter, the eigenstates of $H_{0}$ are the Bloch spinors:

$$
\langle\boldsymbol{r} \sigma \mid \boldsymbol{k} \mu \alpha\rangle=\frac{1}{\sqrt{\mathcal{V}}} \varphi_{\boldsymbol{k} \mu}(\boldsymbol{r}) e^{i \boldsymbol{k r}} \chi_{\alpha}(\sigma)
$$

where $\mathcal{V}$ is the system volume, $\sigma$ is the spin projection, $\varphi_{\boldsymbol{k} \mu}(\boldsymbol{r})$ have the same periodicity as the crystal lattice, and $\chi_{\alpha}$ are the basis spinors: $\chi_{\alpha}(\sigma)=\delta_{\alpha \sigma}$. The Bloch spinors are labelled by the wave vector $\boldsymbol{k}$, the band index $\mu$, and the spin index $\alpha$, which distinguishes the states within the same band. The eigenvalues $\epsilon_{\mu}(\boldsymbol{k})$ describe the electron dispersion in the $\mu$ th band and have the following symmetry properties: $\epsilon_{\mu}(\boldsymbol{k})=\epsilon_{\mu}(-\boldsymbol{k}), \epsilon_{\mu}(\boldsymbol{k})=$ $\epsilon_{\mu}\left(g^{-1} \boldsymbol{k}\right)$, where $g$ is any operation from the point group of the crystal.

Next, we calculate the matrix elements of $H_{S O}$ in the basis of the Bloch states (A2):

$$
\begin{aligned}
\left\langle\boldsymbol{k} \mu \alpha\left|H_{S O}\right| \boldsymbol{p} \nu \beta\right\rangle & =\frac{\hbar^{2}}{4 m^{2} c^{2}} \sum_{i j k} e_{i j k}\left\langle\alpha\left|\hat{\sigma}_{i}\right| \beta\right\rangle \\
& \times \frac{1}{\mathcal{V}} \int d^{3} \boldsymbol{r} \Theta_{j k}(\boldsymbol{r}) e^{i(\boldsymbol{p}-\boldsymbol{k}) \boldsymbol{r}}
\end{aligned}
$$

where $i, j, k=x, y, z$, and

$$
\Theta_{j k}=\left(\nabla_{j} U\right) \varphi_{\boldsymbol{k} \mu}^{*}\left(-i \nabla_{k}+p_{k}\right) \varphi_{\boldsymbol{p} \nu} .
$$

Since $\Theta_{j k}$ are lattice-periodic functions of $\boldsymbol{r}$, the integral in Eq. A3 is nonzero only if $\boldsymbol{p}-\boldsymbol{k}=\boldsymbol{G}$, where $\boldsymbol{G}$ is a reciprocal lattice vector. Because both $\boldsymbol{k}$ and $\boldsymbol{p}$ are in the first Brillouin zone, the only possibility is $\boldsymbol{p}=\boldsymbol{k}$. The Hamiltonian remains a nondiagonal matrix in both the band and spin spaces, and has the following form in the second-quantization representation:

$$
H_{0}=\sum_{\boldsymbol{k}, \mu \nu, \alpha \beta}\left[\epsilon_{\mu}(\boldsymbol{k}) \delta_{\mu \nu} \delta_{\alpha \beta}+\gamma_{\mu \nu}(\boldsymbol{k}) \boldsymbol{\sigma}_{\alpha \beta}\right] a_{\boldsymbol{k} \mu \alpha}^{\dagger} a_{\boldsymbol{k} \nu \beta},
$$

where the chemical potential is included in the band dispersion functions, and

$$
\begin{aligned}
& \gamma_{\mu \nu}(\boldsymbol{k})=\frac{\hbar^{2}}{4 m^{2} c^{2}} \\
& \times \frac{1}{v} \int_{v} d^{3} \boldsymbol{r}\left[(\boldsymbol{\nabla} U) \times \varphi_{\boldsymbol{k} \mu}^{*}(-i \boldsymbol{\nabla}+\boldsymbol{k}) \varphi_{\boldsymbol{k} \nu}\right]
\end{aligned}
$$

(the integration is performed over the unit cell of volume $v)$. The expression (A4) is exact for non-interacting electrons, regardless of the band structure and the strength of the SO coupling.

The functions $\gamma_{\mu \nu}(\boldsymbol{k})$ satisfy certain symmetryimposed conditions. Since $H_{0}$ is Hermitian, we have

$$
\gamma_{\mu \nu}(\boldsymbol{k})=\gamma_{\nu \mu}^{*}(\boldsymbol{k})
$$

Under the point group operations $g$, e.g. rotations, the second-quantization operators transform as follows: $a_{\boldsymbol{k} \mu \alpha}^{\dagger} \rightarrow \sum_{\beta} a_{g \boldsymbol{k}, \mu \beta}^{\dagger} \mathcal{U}_{\beta \alpha}(g)$, where $\hat{\mathcal{U}}(g)=e^{-i \theta(\boldsymbol{n} \hat{\boldsymbol{\sigma}}) / 2}$ is the spinor representation of the rotation about a direction $\boldsymbol{n}$ by an angle $\theta .38$ Requiring that $H_{0}$ remains invariant under $g$ we obtain:

$$
\boldsymbol{\gamma}_{\mu \nu}(\boldsymbol{k})=g \boldsymbol{\gamma}_{\mu \nu}\left(g^{-1} \boldsymbol{k}\right)
$$

Under time reversal, $f a_{\boldsymbol{k} \mu \alpha}^{\dagger} \rightarrow f^{*} \sum_{\beta}\left(i \sigma_{2}\right)_{\alpha \beta} a_{-\boldsymbol{k} \mu \beta}^{\dagger}$ ( $f$ is an arbitrary $c$-number coefficient), therefore $\gamma_{\mu \nu}(\boldsymbol{k}) \rightarrow$ $-\gamma_{\mu \nu}^{*}(-\boldsymbol{k})$. If the time-reversal symmetry is not broken, we have

$$
\gamma_{\mu \nu}(\boldsymbol{k})=-\gamma_{\mu \nu}^{*}(-\boldsymbol{k})
$$

Finally, under inversion $a_{\boldsymbol{k} \mu \alpha}^{\dagger} \rightarrow a_{-\boldsymbol{k} \mu \alpha}^{\dagger}$, and $\gamma_{\mu \nu}$ transform like pseudovectors: $\gamma_{\mu \nu}(\boldsymbol{k}) \rightarrow \gamma_{\mu \nu}(-\boldsymbol{k})$.

In a centrosymmetric crystal, $\gamma_{\mu \nu}(\boldsymbol{k})=\gamma_{\mu \nu}(-\boldsymbol{k})$, therefore, using Eq. (A8),

$$
\gamma_{\mu \nu}(\boldsymbol{k})=-\gamma_{\mu \nu}^{*}(\boldsymbol{k})
$$

It follows from the conditions (A6) and (A9) that $\gamma_{\mu \mu}(\boldsymbol{k})=0$. Therefore one needs to include at least two bands in Eq. (A4), in which case $\gamma_{12}(\boldsymbol{k})=-\boldsymbol{\gamma}_{21}(\boldsymbol{k})=$ $i \boldsymbol{\ell}(\boldsymbol{k})$, and the Hamiltonian takes the form

$$
\begin{aligned}
H_{0}= & \sum_{\boldsymbol{k}, \alpha} \sum_{\mu=1,2} \epsilon_{\mu}(\boldsymbol{k}) a_{\boldsymbol{k} \mu \alpha}^{\dagger} a_{\boldsymbol{k} \mu \alpha} \\
& +i \sum_{\boldsymbol{k}, \alpha \beta} \boldsymbol{\ell}(\boldsymbol{k}) \boldsymbol{\sigma}_{\alpha \beta}\left(a_{\boldsymbol{k} 1 \alpha}^{\dagger} a_{\boldsymbol{k} 2 \beta}-a_{\boldsymbol{k} 2 \alpha}^{\dagger} a_{\boldsymbol{k} 1 \beta}\right)
\end{aligned}
$$


see also Ref. 23. The pseudovector $\boldsymbol{\ell}$ is real, even in $\boldsymbol{k}$, and satisfies $\boldsymbol{\ell}(\boldsymbol{k})=g \boldsymbol{\ell}\left(g^{-1} \boldsymbol{k}\right)$.

In contrast, in a noncentrosymmetric crystal, the constraint (A9) is absent, and the effects of SO coupling can be studied in a minimal model in which one keeps just one band in the Hamiltonian (A4). The band index $\mu$ can then be dropped and the SO coupling can be de- scribed by a single pseudovector function $\gamma(\boldsymbol{k})$, which is real, odd in $\boldsymbol{k}$, and invariant with respect to the point group operations: $\boldsymbol{\gamma}(\boldsymbol{k})=g \boldsymbol{\gamma}\left(g^{-1} \boldsymbol{k}\right)$. In this way one arrives at the effective band Hamiltonian (1). Although in principle one can calculate $\gamma(\boldsymbol{k})$ using Eq. (A5), we consider it as a model parameter.
1 E. Bauer, G. Hilscher, H. Michor, Ch. Paul, E. W. Scheidt, A. Gribanov, Yu. Seropegin, H. Noël, M. Sigrist, and P. Rogl, Phys. Rev. Lett. 92, 027003 (2004).

2 T. Akazawa, H. Hidaka, T. Fujiwara, T. C. Kobayashi, E. Yamamoto, Y. Haga, R. Settai, and Y. Onuki, J. Phys.: Condens. Matter 16, L29 (2004).

3 N. Kimura, K. Ito, K. Saitoh, Y. Umeda, H. Aoki, T. Terashima, Phys. Rev. Lett. 95, 247004 (2005).

4 I. Sugitani, Y. Okuda, H. Shishido, T. Yamada, A. Thamizhavel, E. Yamamoto, T. D. Matsuda, Y. Haga, T. Takeuchi, R. Settai, and Y. Onuki, J. Phys. Soc. Jpn. 75, 043703 (2006).

${ }^{5}$ G. Amano, S. Akutagawa, T. Muranaka, Y. Zenitani, and J. Akimitsu, J. Phys. Soc. Jpn 73, 530 (2004).

${ }^{6}$ K. Togano, P. Badica, Y. Nakamori, S. Orimo, H. Takeya, and K. Hirata, Phys. Rev. Lett. 93, 247004 (2004); P. Badica, T. Kondo, and K. Togano, J. Phys. Soc. Jpn. 74, 1014 (2005).

7 K. V. Samokhin, E. S. Zijlstra, and S. K. Bose, Phys. Rev. B 69, 094514 (2004) [Erratum: 70, 069902(E) (2004)].

${ }^{8}$ K.-W. Lee and W. E. Pickett, Phys. Rev. B 72, 174505 (2005).

9 L. P. Gor'kov and E. I. Rashba, Phys. Rev. Lett. 87, 037004 (2001).

10 I. A. Sergienko and S. H. Curnoe, Phys. Rev. B 70, 214510 (2004).

11 V. P. Mineev, Int. J. Mod. Phys. B 18, 2963 (2004).

12 V. P. Mineev and K. V. Samokhin, preprint cond-mat/0612546.

13 V. M. Edelstein, Zh. Eksp. Teor. Fiz. 95, 2151 (1989) [Sov. Phys. JETP 68, 1244 (1989)].

14 P. A. Frigeri, D. F. Agterberg, A. Koga, and M. Sigrist, Phys. Rev. Lett. 92, 097001 (2004) [Erratum 93, 099903(E) (2004)].

15 P. A. Frigeri, D. F. Agterberg, I. Milat, and M. Sigrist, preprint cond-mat/0505108.

16 L. N. Bulaevskii, A. A. Guseinov, and A. I. Rusinov, Zh. Eksp. Teor. Fiz. 71, 2356 (1976) [Sov. Phys. JETP 44, 1243 (1976)].

17 S. K. Yip, Phys. Rev. B 65, 144508 (2002).

18 P. A. Frigeri, D. F. Agterberg, and M. Sigrist, New J. Phys. 6, 115 (2004).

19 K. V. Samokhin, Phys. Rev. Lett. 94, 027004 (2005).

20 S. Fujimoto, preprint cond-mat/0605290

${ }^{21}$ M. Yogi, H. Mukuda, Y. Kitaoka, S. Hashimoto, T. Ya- suda, R. Settai, T. D. Matsuda, Y. Haga, Y. Onuki, P. Rogl, and E. Bauer, J. Phys. Soc. Jpn. 75, 013709 (2005).

22 W. A. Hines and W. D. Knight, Phys. Rev. B 4, 893 (1971).

23 L. P. Gor'kov, Zh. Eksp. Teor. Fiz. 48, 1772 (1965) [Sov. Phys. JETP 21, 1186 (1965)].

24 R. A. Ferrell, Phys. Rev. Lett. 3, 262 (1959).

25 P. W. Anderson, Phys. Rev. Lett. 3, 325 (1959).

26 A. A. Abrikosov and L. P. Gor'kov, Zh. Eksp. Teor. Fiz. 42, 1088 (1962) [Sov. Phys. JETP 15, 752 (1962)].

27 E. I. Rashba, Fiz. Tverd. Tela (Leningrad) 2, 1224 (1960) [Sov. Phys. Solid State 2, 1109 (1960)].

28 V. P. Mineev and K. V. Samokhin, Introduction to Unconventional Superconductivity (Gordon and Breach, London, 1999).

29 A. A. Abrikosov, L. P. Gorkov, and I. E. Dzyaloshinski, Methods of Quantum Field Theory in Statistical Physics (Dover, New York, 1975).

30 The origin of the negative signs in Eqs. (35) and (36) can be traced back to our definition of the time reversal operator: $K=i \hat{\sigma}_{2} K_{0}$ ( $K_{0}$ is the complex conjugation). If one uses $K=-i \hat{\sigma}_{2} K_{0}$ instead, then $\psi=\left(\tilde{\Delta}_{+}+\tilde{\Delta}_{-}\right) / 2$ and $\boldsymbol{d}=$ $\left(\tilde{\Delta}_{+}-\tilde{\Delta}_{-}\right) \hat{\gamma} / 2$, as in Ref. 11 .

31 V. P. Mineev, P. L. Krotkov, Phys. Rev. B 65, 024501 (2001).

32 P. Sharma and J. A. Sauls, J. Low Temp. Phys. 125, 115 (2001).

33 T. Yasuda, H. Shishido, T. Ueda, S. Hashimoto, R. Settai, T. Takeuchi, T. D. Matsuda, Y. Haga, and Y. Onuki, J. Phys. Soc. Jpn. 73, 1657 (2004).

34 M. Yogi, Y. Kitaoka, S. Hashimoto, T. Yasuda, R. Settai, T. D. Matsuda, Y. Haga, Y. Onuki, P. Rogl, and E. Bauer, Phys. Rev. Lett. 93, 027003 (2004).

35 K. Izawa, Y. Kasahara, Y. Matsuda, K. Behnia, T. Yasuda, R. Settai, and Y. Onuki, Phys. Rev. Lett. 94, 197002 (2005).

36 I. Bonalde, W. Bramer-Escamilla, and E. Bauer, Phys. Rev. Lett. 94, 207002 (2005).

37 N. Tateiwa, Y. Haga, T. D. Matsuda, S. Ikeda, T. Yasuda, T. Takeuchi, R. Settai, and Y. Onuki, J. Phys. Soc. Jpn. 74, 1903 (2005).

38 The transformation rules for the creation and annihilation operators are discussed, e.g. in J. P. Elliott and P. G. Dawber, Symmetry in Physics, Vol. 2, Ch. 16 (McMillan Press, London, 1979). 